\title{
Altered visual and haptic verticality perception in posterior cortical atrophy and Alzheimer's disease
}

Brian L. Day ${ }^{1}$, Dilek Ocal ${ }^{2,3}$, Amy Peters ${ }^{1}$, Matthew J. Bancroft ${ }^{1}$ (D), David Cash ${ }^{2}$, Diego Kaski $^{1}$, Sebastian J. Crutch ${ }^{2}$ and Keir X. X. Yong ${ }^{2}$ (D)

${ }^{1}$ Department of Clinical and Movement Neurosciences, UCL Queen Square Institute of Neurology, London, UK

${ }^{2}$ Dementia Research Centre, Department of Neurodegenerative Disease, UCL Queen Square Institute of Neurology, University College London, London, $U K$

${ }^{3}$ Department of Neurophysics, Max Planck Institute for Human Cognitive and Brain Sciences, Leipzig, DE

Edited by: Richard Carson \& Vaughan Macefield

The peer review history is available in the Supporting Information section of this article (https://doi.org/10.1113/JP282289\#support-information-section).

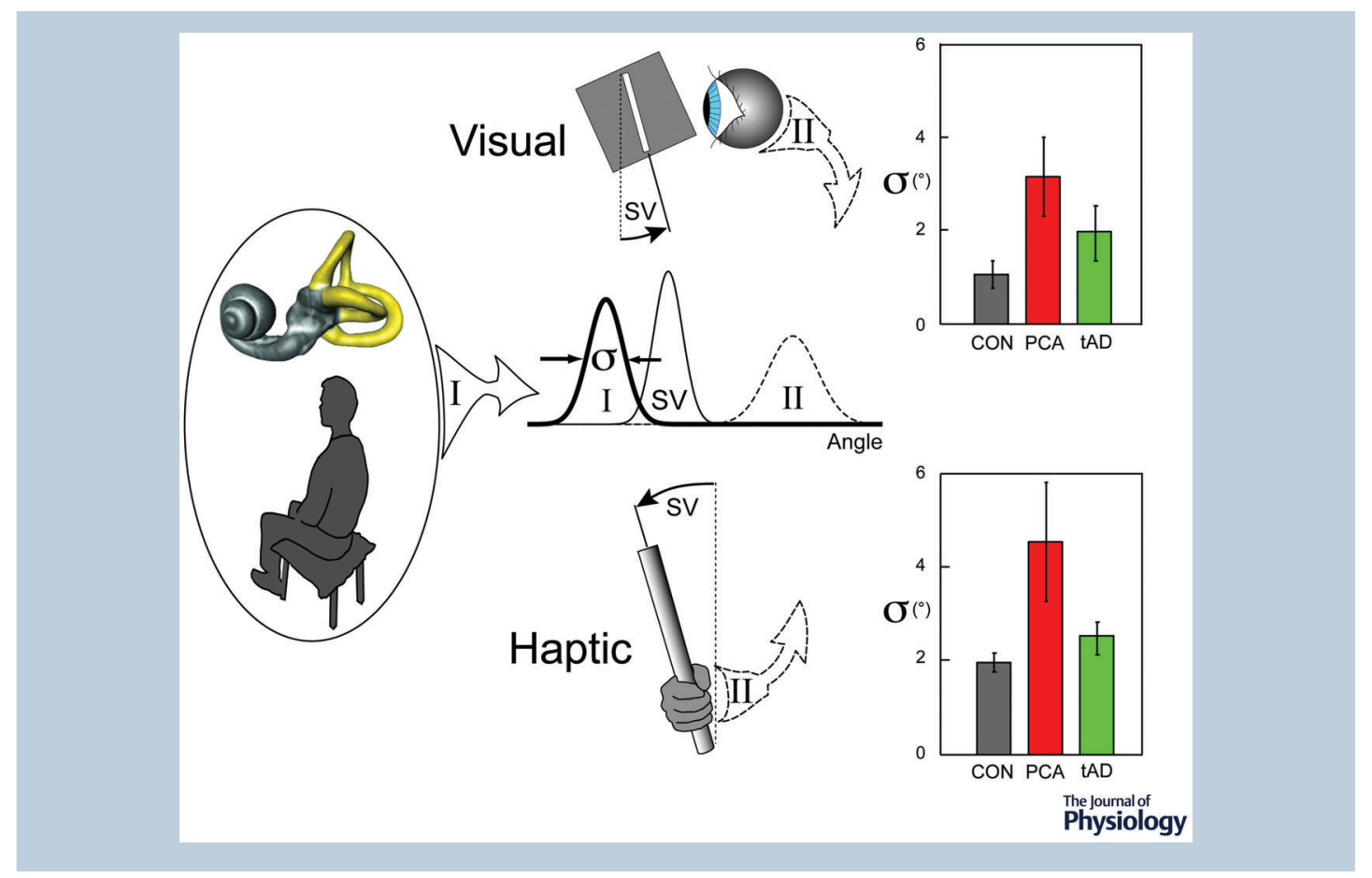

Brian Day is Emeritus Professor of Motor Neuroscience at University College London. His work has encompassed many aspects of human sensorimotor processes, both in health and in a wide range of neurological diseases. He has been at the forefront in the development and application of non-invasive techniques for studying central nervous system function and circuitry in intact man, most notably transcranial magnetic and electric stimulation, and more recently vestibular stimulation. His current research is devoted to understanding multi-sensory processes that control and integrate whole-body action. 


\begin{abstract}
There is increasing theoretical and empirical support for the brain combining multisensory information to determine the direction of gravity and hence uprightness. A fundamental part of the process is the spatial transformation of sensory signals between reference frames: eye-centred, head-centred, body-centred, etc. The question 'Am I the right way up?' posed by a patient with posterior cortical atrophy (PCA) suggests disturbances in upright perception, subsequently investigated in PCA and typical Alzheimer's disease (tAD) based on what looks or feels upright. Participants repeatedly aligned to vertical a rod presented either visually (visual-vertical) or haptically (haptic-vertical). Visual-vertical involved orienting a projected rod presented without or with a visual orientation cue (circle, tilted square $\left( \pm 18^{\circ}\right)$ ). Haptic-vertical involved orientating a grasped rod with eyes closed using a combination of side (left, right) and hand (unimanual, bimanual) configurations. Intraindividual uncertainty and bias defined verticality perception. Uncertainty was consistently greater in both patient groups than in control groups, and greater in PCA than tAD. Bias in the frontal plane was strongly directionally affected by visual cue tilt (visual-vertical) and grip side (haptic-vertical). A model was developed that assumed verticality information from multiple sources is combined in a statistically optimal way to produce observed uncertainties and biases. Model results suggest the mechanism that spatially transforms graviceptive information between body parts is disturbed in both patient groups. Despite visual dysfunction being typically considered the primary feature of PCA, disturbances were greater in PCA than tAD particularly for haptic-vertical, and are considered in light of posterior parietal vulnerability.
\end{abstract}

(Received 23 August 2021; accepted after revision 26 November 2021; first published online 28 November 2021)

Corresponding author K. Yong: Dementia Research Centre, National Hospital for Neurology and Neurosurgery, Box 16, Queen Square, London WC1N 3BG, UK. Email: keir.yong@ucl.ac.uk

\begin{abstract}
Participants with posterior cortical atrophy (PCA), typical Alzheimer's disease (tAD) or controls $(\mathrm{CON})$ repeatedly aligned to vertical a rod presented visually (visual-vertical, top) or haptically in the absence of visual feedback (haptic-vertical, bottom). For all tasks, participants' subjective verticality estimation (SV) was defined by observed perceptual bias (mean) and uncertainty (SD). A mechanistic model was developed based on principles of maximum likelihood estimation (Gaussians, centre) to estimate the uncertainty $(\sigma)$ associated with a primary mechanism which spatially transforms information from a system of graviceptors using information about whole-body configuration (I, left). SV was modelled as the product of the primary mechanism (I) and independent local secondary mechanisms (II; retinal-based or hand-based for visual and haptic tasks, respectively). Conservative estimates of primary-mechanism uncertainty $(\sigma)$ were greatest in PCA relative to tAD and control groups across tasks (right; estimated means and 95\% CI), being particularly apparent for the haptic-vertical task.
\end{abstract}

\title{
Key points
}

- The perception of upright requires accurate and precise estimates of orientation based on multiple noisy sensory signals.

- The question 'Am I the right way up?' posed by a patient with posterior cortical atrophy (PCA; purported 'visual variant Alzheimer's') suggests disturbances in the perception of upright.

- What looks or feels upright in PCA and typical Alzheimer's disease (tAD) was investigated by asking participants to repeatedly align to vertical a rod presented visually (visual-vertical) or haptically (haptic-vertical).

- PCA and tAD groups exhibited not only greater perceptual uncertainty than controls, but also exaggerated bias induced by tilted visual orientation cues (visual-vertical) and grip side (haptic-vertical). When modelled, these abnormalities, which were particularly evident in PCA haptic-vertical performance, were compatible with disruption of a mechanism that spatially transforms verticality information between body parts.

- The findings suggest an important role of posterior parietal cortex in verticality perception, and have implications for understanding spatial disorientation in dementia. 


\section{Introduction}

A good sense of verticality requires accurate and precise estimates of orientation relative to gravity predicated on multiple noisy sensory signals. The noisy sensory signals undergo spatial transformations from the egocentric reference frame of the body part in which the sensory receptors are embedded (e.g. eye-, head- and body-centred) into an allocentric, gravity-centred reference frame (Mittelstaedt, 1983; Andersen et al. 1985; Dakin \& Rosenberg, 2018). The posterior parietal lobes are key candidate anatomical loci for such multisensory integration and reference frame transformations (Duhamel et al. 1998; Buneo et al. 2002), and so diseases that affect parietal regions might be expected to distort the sense of verticality and produce vertical-plane disorientation. Disorientation in the vertical plane can manifest as compromised postural or locomotor functions as well as perceptual deficits. Therefore, parietal vulnerability in Alzheimer's disease (AD) may contribute to the perceptual disturbances, disorders of gait, balance and posture, increased falls incidence, and spatial disorientation that have been reported (Allan et al. 2009; Golden et al. 2015; Coughlan et al. 2018; Hardy et al. 2020; Van Engelen et al. 2020).

To investigate this hypothesis, we have studied vertical-plane disorientation by measuring verticality perception in two groups of patients with neurodegenerative disease varying in clinical presentation. One patient group presented with typical, memory-led Alzheimer's disease (tAD), the other with posterior cortical atrophy (PCA), often considered the most common atypical AD clinical phenotype (Alladi et al. 2007; Warren et al. 2012; Graff-Radford et al. 2021). Despite most commonly being underpinned by $\mathrm{AD}$ pathology, PCA is characterised by progressive decline in higher visual processing and other posterior cortical functions with relative sparing of memory, language and executive functions (Tang-Wai et al. 2004; Crutch et al. 2017).

While diminished visual functions are considered the most well-recognised or defining features of PCA, patient reports and formal investigations increasingly suggest non-visual perceptual disturbances (Golden et al. 2015; Crutch et al. 2018, 2020; Hardy et al. 2020). Reports suggestive of abnormal multisensory integration, vertical-plane disorientation and subjective balance disorder include PCA patients asking 'am I the right way up?' while sitting, commenting 'I felt like I was about to fall off the edge of the world' when walking, and noting sensations of 'floating along the ceiling' after descending stairs (Crutch et al. 2018). Postural disturbances such as leaning to one side during walking or standing have been observed in a number of PCA and late stage young-onset AD patients (Crutch et al. 2018; Van Engelen et al. 2020) consistent with disorientation in the vertical plane. In PCA relative to $\mathrm{tAD}$, neuroanatomical differences comprise reduced grey matter volume in posterior parietal and occipito-temporal regions and relative preservation of medial temporal, hippocampal and entorhinal regions (Whitwell et al. 2007; Lehmann et al. 2011; Firth et al. 2019). We predicted, therefore, that verticality perception would be disturbed in both patient groups relative to controls, but more so in PCA than tAD based on the greater vulnerability of parietal cortices in PCA.

Two broad tests of verticality perception were employed. In the first, participants were asked to orient a visually presented rod to Earth vertical with or without distorting visual orientation cues. In the second, participants were asked to orient a manually held rod to Earth vertical without visual feedback, under varying haptic feedback (grip) conditions. The rationale was that a disorder of verticality should manifest across both tests even in the absence of visual feedback, despite visual disturbances being characteristically associated with PCA (purported 'visual variant AD'). We measured perceptual bias (inaccuracy) and uncertainty (imprecision) of verticality perception, and used these to model the degree of disruption to spatial transformation of sensory information from egocentric to allocentric reference frames.

\section{Methods}

\section{Ethical approval}

Informed consent was obtained from all participants. The study conformed to standards set by the Declaration of Helsinki, with the exception of registration in a public database (World Medical Association, 2013). Prior ethical approval for the study was provided by the National Research Ethics Service Committee London Queen Square (reference: 06/Q0512/81).

\section{Participants}

Twenty-two PCA patients, 21 tAD patients and 21 healthy controls were included in the study. PCA and tAD groups fulfilled clinical criteria for PCA-pure and research criteria for probable $\mathrm{AD}$, respectively (McKhann et al. 2011; Crutch et al. 2017). Groups were of comparable sex and patient groups were of comparable disease severity (Table 1). Molecular pathology (amyloid imaging performed as part of another investigation or cerebrospinal fluid (CSF)) was available for 6/22 PCA and $3 / 21$ tAD patients; all were consistent with $\mathrm{AD}$ pathology. The PCA group was younger than both $\mathrm{tAD}$ and control groups; the effect of age on task performance was subsequently investigated ('Statistical methods'). 
See Table 1 for vibrotactile sensitivity, neuropsychological scores and estimated performance relative to normative datasets for PCA and tAD groups. Background neuropsychological assessment and group comparison of atrophy profiles using voxel-based morphometry emphasised space and object perceptual deficits and parietal atrophy in PCA relative to tAD (Fig. 1). Consistent with clinical diagnosis, corticovisual impairments were evident in all PCA patients in contrast to relatively spared episodic memory and language. The neuropsychological profile of tAD patients overall was consistent with an episodic memory and language-led presentation, with a minority exhibiting corticovisual impairments.

\section{Sensory and neuro-otological assessment}

Biothesiometer ascending threshold measures of vibrotactile sensitivity were assessed over four sites

Table 1. Demographic information and PCA and tAD neuropsychological scores

\begin{tabular}{|c|c|c|c|c|c|}
\hline A. Demographic information & \multicolumn{2}{|c|}{ PCA $(n=22)$} & \multicolumn{2}{|c|}{$\mathrm{tAD}(n=21)$} & $\begin{array}{l}\text { Control } \\
(n=21)\end{array}$ \\
\hline Sex (male:female) & \multicolumn{2}{|c|}{ 11:11 } & \multicolumn{2}{|c|}{$12: 9$} & $13: 8$ \\
\hline Age* $^{*}$ & \multicolumn{2}{|c|}{$65.4 \pm 7.1$} & \multicolumn{2}{|c|}{$73.0 \pm 7.2$} & $70.3 \pm 5.2$ \\
\hline MMSE $^{a}$ & \multicolumn{2}{|c|}{$23.2 \pm 4.0$} & \multicolumn{2}{|c|}{$21.9 \pm 5.4$} & - \\
\hline$\beta$-Amyloid PET/CSF consistent with $\mathrm{AD}^{\dagger}$ & \multicolumn{2}{|c|}{$6 / 6$} & \multicolumn{2}{|c|}{$3 / 3$} & \\
\hline \multicolumn{6}{|l|}{ Vibration threshold $(\mu \mathrm{m})^{\ddagger}$} \\
\hline Plantar hallux & \multicolumn{2}{|c|}{$19.2 \pm 7.2$} & \multicolumn{2}{|c|}{$28.5 \pm 12.0$} & $25.3 \pm 12.1$ \\
\hline Plantar metatarsal head & \multicolumn{2}{|c|}{$17.6 \pm 9.2$} & \multicolumn{2}{|c|}{$27.6 \pm 13.0$} & $26.1 \pm 14.1$ \\
\hline Plantar heel & \multicolumn{2}{|c|}{$16.7 \pm 8.1$} & \multicolumn{2}{|c|}{$28.1 \pm 11.5$} & $26.9 \pm 14.2$ \\
\hline \multirow[t]{2}{*}{ Mid-tibia } & \multicolumn{2}{|c|}{$27.4 \pm 12.1$} & \multicolumn{2}{|c|}{$31.9 \pm 10.3$} & $30.0 \pm 10.4$ \\
\hline & & \multicolumn{2}{|c|}{ Raw score } & \multicolumn{2}{|c|}{$\begin{array}{l}\% \text { patients below } 5 \text { th } \\
\text { percentile }\end{array}$} \\
\hline B. Neuropsychology test & Max & PCA & tAD & PCA & tAD \\
\hline \multicolumn{6}{|l|}{ Background neuropsychology } \\
\hline $\begin{array}{l}\text { Short recognition memory test for words (joint } \\
\text { auditory/visual presentation) }\end{array}$ & 25 & $21.4 \pm 3.0$ & $18.2 \pm 3.7$ & $3 / 19(16 \%)$ & $9 / 20(45 \%)$ \\
\hline Concrete synonyms test & 25 & $20.8 \pm 2.7$ & $20.6 \pm 3.2$ & $3 / 19(16 \%)$ & $4 / 20(20 \%)$ \\
\hline Naming (verbal description) & 20 & $16.4 \pm 4.0$ & $14.4 \pm 4.9$ & $7 / 19(37 \%)$ & $11 / 20(55 \%)$ \\
\hline Calculation $\left(\mathrm{GDA}^{\mathrm{b}}\right)$ & 24 & $4.8 \pm 5.3$ & $8.2 \pm 8.5$ & $7 / 13(54 \%)$ & $9 / 18(50 \%)$ \\
\hline Spelling (GDST ${ }^{\mathrm{C}}$ set $\mathrm{B}$, first 20 items) & 20 & $12.7 \pm 6.5$ & $12.6 \pm 6.3$ & $4 / 19(21 \%)$ & $3 / 19(16 \%)$ \\
\hline Gesture production test & 15 & $13.7 \pm 1.8$ & $14.9 \pm 0.4$ & & \\
\hline Digit span (forwards) & 12 & $6.3 \pm 2.7$ & $6.8 \pm 1.8$ & $5 / 19(26 \%)$ & $2 / 20(10 \%)$ \\
\hline Max forwards & 8 & $5.3 \pm 1.6$ & $5.7 \pm 1.0$ & & \\
\hline Digit span (backwards) & 12 & $3.5 \pm 2.0$ & $4.0 \pm 2.5$ & $3 / 18(28 \%)$ & $5 / 20(25 \%)$ \\
\hline Max backwards & 7 & $2.7 \pm 1.4$ & $3.3 \pm 1.5$ & & \\
\hline \multicolumn{6}{|l|}{ Visual assessment } \\
\hline \multicolumn{6}{|l|}{ Early visual } \\
\hline Visual acuity (CORVIST'): Snellen (median) & $6 / 9$ & $6 / 9$ & $6 / 9$ & - & - \\
\hline Figure-ground discrimination (VOSPe) & 20 & $17.0 \pm 2.1$ & $19.1 \pm 0.8$ & $14 / 19(74 \%)$ & $5 / 20(25 \%)$ \\
\hline Shape discrimination ${ }^{f}$ & 20 & $14.6 \pm 4.2$ & $18.3 \pm 3.2$ & $\begin{array}{l}\text { Healthy } \\
\text { participants } \\
\text { do not } \\
\text { make } \\
\text { errors }\end{array}$ & \\
\hline \multicolumn{6}{|l|}{ Visuoperceptual } \\
\hline Fragmented letters (VOSP) & 20 & $10.1 \pm 5.5$ & $16.7 \pm 5.3$ & $16 / 18(89 \%)$ & $4 / 20(20 \%)$ \\
\hline Object decision (VOSP) & 20 & $11.5 \pm 4.5$ & $16.7 \pm 2.6$ & $13 / 18(72 \%)$ & $4 / 20(20 \%)$ \\
\hline Unusual and usual views: unusual & 20 & $4.8 \pm 3.8$ & $12.6 \pm 4.3$ & $18 / 18(100 \%)$ & $9 / 20(45 \%)$ \\
\hline Usual & 20 & $15.8 \pm 4.7$ & $18.8 \pm 2.0$ & $14 / 18(78 \%)$ & $4 / 20(20 \%)$ \\
\hline
\end{tabular}


Table 1. (Continued)

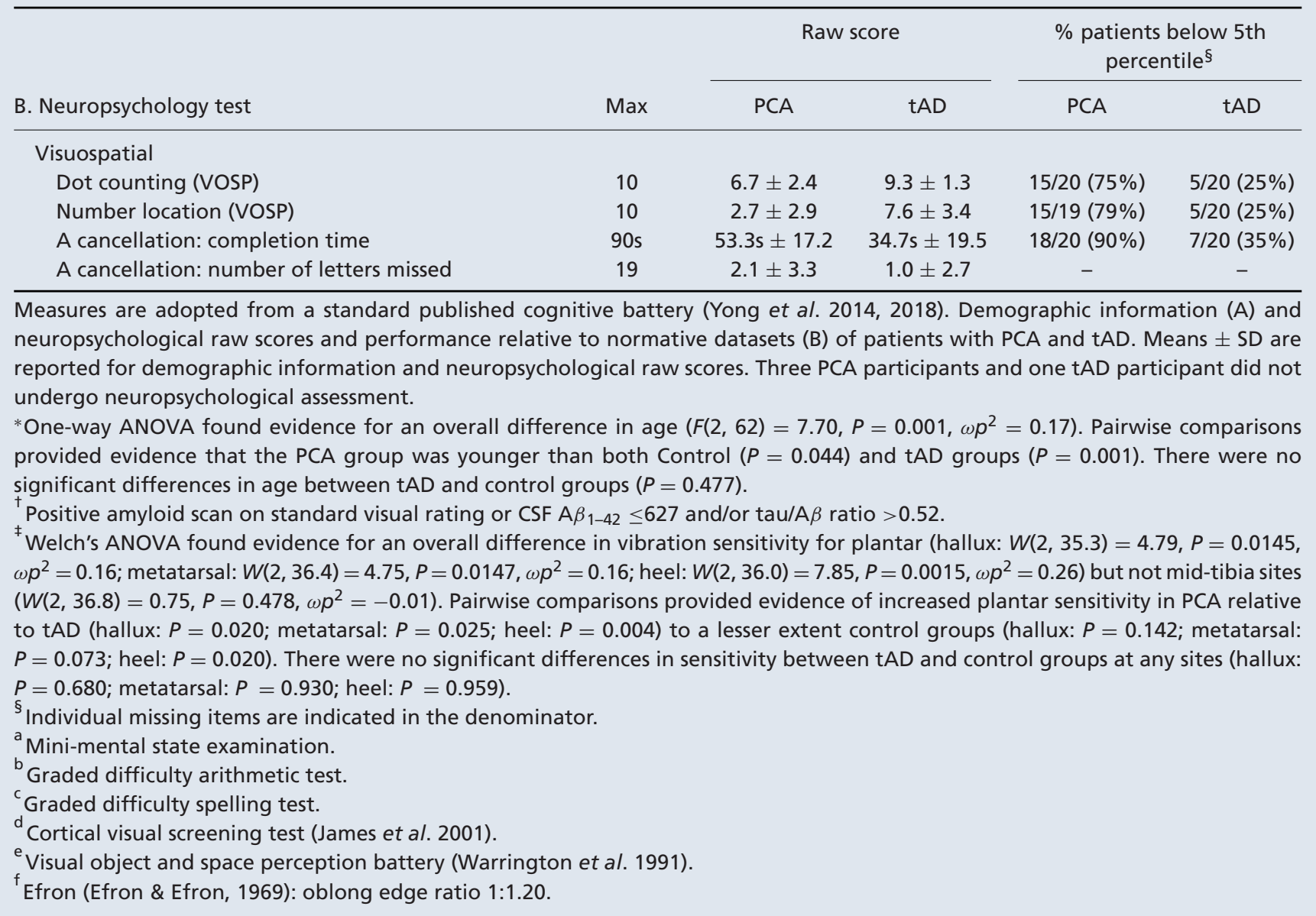

on both lower limbs (plantar hallux, plantar metatarsal head, plantar heel and mid-tibia) for a total of eight sites. Sensitivity was assessed three times at each site, with recordings combined across sides to provide four medians per participant. There was no evidence of decreased vibration sensitivity in either PCA or tAD relative to the control group (Table 1). All participants underwent a detailed clinical neurological history to exclude underlying ophthalmological, vestibular, psychiatric or other neurological diseases.

Neuro-otological clinical assessments were conducted in a subset of participants (PCA: 19/22; tAD: 11/21) comprising ophthalmoscopy to exclude ocular disorders, oculomotor assessments (fixation, saccades, spontaneous and gaze-evoked nystagmus, pursuit, vestibulo-ocular reflex testing, and positional manoeuvres), gait assessment and the Romberg test. Clinical vestibular assessments were normal in all patients assessed. While clear oculomotor abnormalities were not evident on clinical assessment, subtle oculomotor changes were noted in the PCA group, including square wave jerks, increased saccadic latency and saccadic hypometria. Oculomotor assessment was normal in patients with $\mathrm{tAD}$ apart from the presence of square wave jerks on fixation. Oculomotor abnormalities were in line with previous investigations and consistent with core features of PCA and Balint's syndrome (oculomotor apraxia) (Mendez et al. 2002; Shakespeare et al. 2015; Crutch et al. 2017). One healthy control was excluded owing to positional downbeat nystagmus.

\section{Procedures}

Two tests of verticality perception were performed on separate days. Each tested the ability to align a rod with Earth vertical, first using an image of a rod projected on a screen (visual vertical; VV), and secondly using a physical rod held manually with eyes closed (haptic vertical; HV).

Both visual-vertical and haptic-vertical tests were preceded by a training period to ensure comprehension of the concept of 'vertical'. If this was not clear a form of words was sought, such as 'upright' or 'straight up and down', which the participant understood to mean vertical 
and which was then subsequently used for instructions. Testing apparatus and procedures were informed by patient feedback from pilot testing (Crutch et al. 2018).

Visual-vertical. Participants were seated in a darkened room with their head held in a fixed upright and forward-facing position by a chair-mounted head-clamp. They faced a floor-to-ceiling $2.4 \mathrm{~m}$-wide projection screen (The Widescreen Centre Ltd, London, UK) at a distance of $600 \mathrm{~mm}$ from the eyes. Images were rear-projected and centred on the participant's head. A $40 \mathrm{~mm}$ diameter knob was fixed to a floor-mounted stand and was positioned close to the participant's preferred hand so it could be easily grasped and turned between thumb and index finger. Visual cues of verticality were occluded using black cloth drapes outside the screen and over the participant's lap and chair.

Projected images (Fig. 2A) were created using LabVIEW (National Instruments, Austin, TX, USA) and consisted of a white rod (length $0.24 \mathrm{~m}$ ) on a grey-filled background shape (circle (diameter $0.26 \mathrm{~m}$ ) or tilted square (side length $0.26 \mathrm{~m}$ )). The projected image outside the rod and grey-filled background was stationary and uniformly black; the background grey shape was stationary but the white rod could be rotated in the frontal plane by the participant turning the knob. In previous work (Dakin et al. 2018) we had created a rod consisting of a yoked linear sequence of separate white dots, but in pilot studies we found that PCA participants sometimes experienced perceptual difficulties combining the yoked dots into a single object. The rod was cylindrical and virtually illuminated in the software. This gave the rod a 3-D appearance with its whiteness tapering to grey at the edges and thus blending with the grey background shape and minimising pixilation cues regarding rod orientation. The image outside of the rod and the grey background shape was uniformly black with stray projector light being excluded using an out-of-focus mask surrounding the intended image. The background grey shape was stationary but the white rod could be rotated in the frontal plane by the participant turning the knob which was connected to a 10-turn potentiometer. The varying potentiometer output voltage was sampled at $33 \mathrm{~Hz}$ by the custom software that was used to generate the image and converted to define rod orientation in real time. Prior to testing, participants were allowed to practice turning the knob to adjust the rod orientation against a grey circle background and to comfortably position their hand and arm.

For each trial, the background shape was either a grey circle, or a grey square tilted $18^{\circ}$ clockwise or counterclockwise. The rod was always initially tilted by $45^{\circ}$ from vertical, either clockwise or counterclockwise with equal probability. Eight trials of each of the three background shapes were presented in pseudo-random order with a black screen between trials to allow time for afterimages to decay, for a total of 24 trials. At the beginning of each trial, participants were instructed to make the rod appear vertical on the screen by turning the knob. They were allowed as much time as they wished, and they indicated to the experimenter verbally once they were satisfied that the rod was vertical, whereupon the rod angle was recorded and the image removed.

Haptic-vertical. Participants were seated on an armless chair and held a wooden cylindrical rod (length $540 \mathrm{~mm}$; diameter $28 \mathrm{~mm}$ ). A thin aluminium rectangular platform $(150 \times 100 \times 1 \mathrm{~mm})$ was fixed to the top of the rod and orthogonal to its long-axis. Using a motion-capture system (Coda, Charnwood Dynamics, Rothley, UK), the

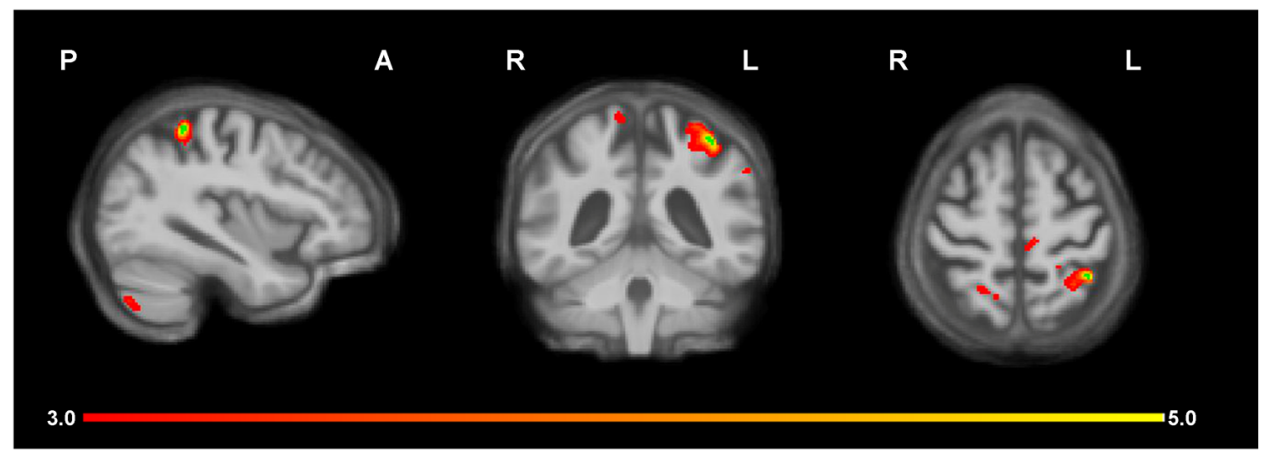

Figure 1. Grey matter differences between PCA and tAD

Whole brain VBM results showing regional variations in grey matter volume in PCA compared to tAD group with available MR scans (PCA $n=14 ; \operatorname{tAD} n=15$ ). The colour bar represents pseudo $t$-scores of increased grey matter volume in the $T A D$ group. Regions where the findings survived family wise error (FWE) correction for multiple comparisons through permutation based peak-voxel inference are shown in green. Group comparison showed reduced left superior posterior parietal grey matter volume in the PCA relative to the TAD group (FWE corrected, $P=0.0276$ ). There were no findings which survived FWE correction using the reverse contrast (i.e. lower grey matter in the TAD relative to the PCA group). 
3-D motion of four markers fixed to each corner of the plate was tracked thereby enabling orientation of the rod's long-axis in space to be measured.

Before each trial, participants were instructed to grip and raise the rod and given a demonstration of the task by

\section{$A$}

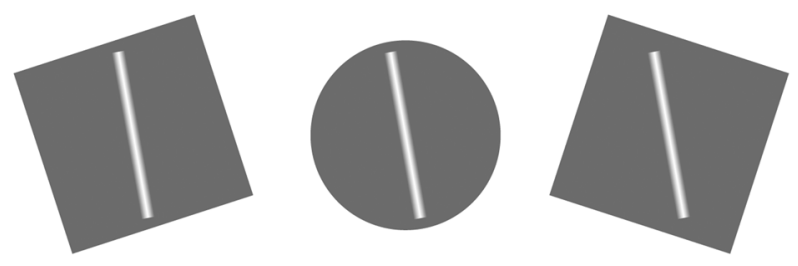

$B$

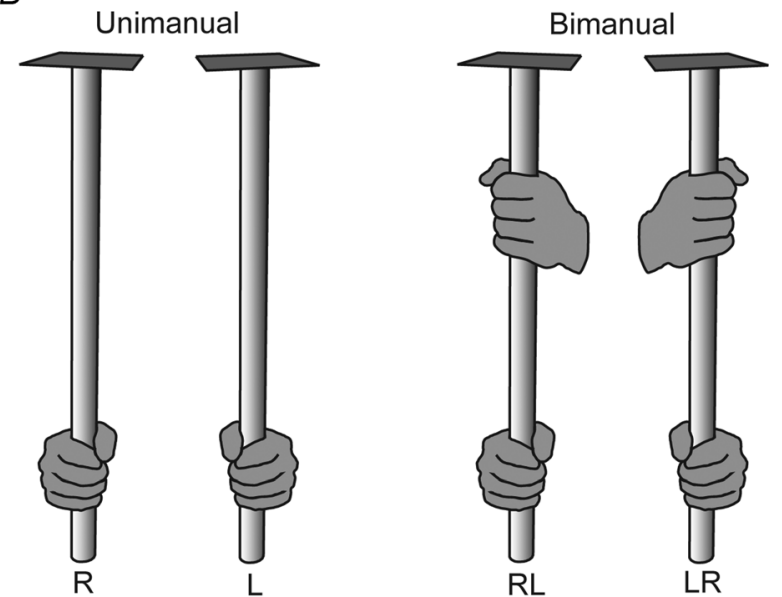

C

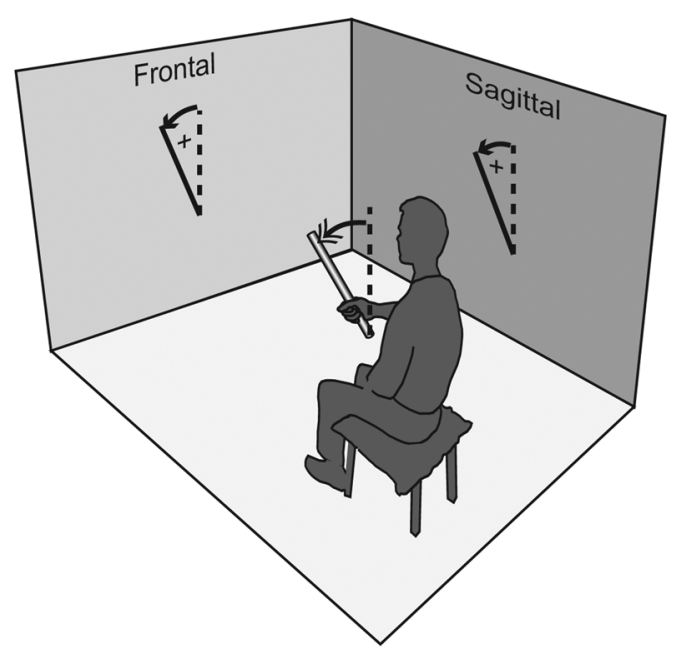

Figure 2. Tests of verticality perception

$A$, visual-vertical $(\mathrm{VV})$ with three background grey shapes: square tilted $18^{\circ}$ counter clockwise; circle; square tilted $18^{\circ}$ clockwise. $B$, haptic-vertical (HV) with four grips: unimanual right hand (R); unimanual left hand $(\mathrm{L})$; bimanual right hand bottom, left hand top $(\mathrm{RL})$; bimanual left hand bottom, right hand top (LR). C, measures of verticality estimation for $\mathrm{HV}$ from projections onto the frontal (as for V) and sagittal planes. the examiner to aid comprehension. The trial proceeded once the examiner was convinced that the participant understood the upcoming task.

Each trial started with the rod being gripped according to instructions and resting horizontally on the participant's thighs. The participant was asked to close their eyes and data collection started and lasted for $1 \mathrm{~min}$. During this period the examiner, who was sat directly in front of the participant and checked eye closure, would give the instruction to raise the rod and make it vertical'. There was no time constraint on this action. Once the examiner judged that the rod was being held in a stationary position, the participant was instructed to 'put the rod down'. If necessary, the examiner would guide the participant's hands to bring the rod back to rest on their thighs. This procedure was repeated a variable number of times depending on the participant's speed until $1 \mathrm{~min}$ of data collection was completed. The participant was then asked to open their eyes and rest for a minute or so whereupon the whole procedure was repeated using a different grip. The procedure was performed under four conditions using a fixed order of grip $(R, L, R L, L R$; Fig. $2 B$ ) for a total of 4 min of data collection (median (IQR) number of total rod lifts per participant: CON, 24 (6); PCA, 22.5 (4.25); tAD, 24 (4.5). The haptic-vertical task was not completed by four participants (two PCA; two tAD) owing to time constraints.

\section{Measures}

Repeated rod alignment trials enabled estimates of perceptual bias (mean rod angle) and perceptual uncertainty (standard deviation rod angle) of verticality for each participant.

Visual-vertical. Without visual orientation cues (grey circle background), $\mathrm{VV}$ bias corresponded to intra-individual mean rod angle with circle background, equivalent to each participant's directional constant error or bias. VV magnitude bias was equivalent to unsigned VV bias, the participant's magnitude of constant error or bias regardless of direction. VVframe bias corresponded to intra-individual mean rod angle with orientation cues (tilted $\left(18^{\circ}\right)$ grey square background). VVframe-directional bias was equivalent to the average frame-induced bias relative to the direction of frame tilt:

((VVframe bias with left tilt - VVframe bias with right tilt) /2)

Averaging over tilt conditions cancelled out any underlying frame-unrelated constant VV bias. VV uncertainty measures were defined for VV bias and VVframe bias corresponding to intra-individual standard deviation across trials (VV uncertainty; VVframe uncertainty). 
Haptic-vertical. HV directional bias was defined as mean angle of the rod projected onto the participant's two-dimensional sagittal (HV sagittal bias) or frontal plane (HV frontal bias). The angle is signed and represents a constant tilt bias of the rod in either sagittal or frontal plane. HVside-directional bias was analogous to VVframe-directional bias and measures the average constant bias relative to the side of the body determining the grip (bottom right or left hand; Fig. $2 B$ ) in the frontal plane, with any underlying side-unrelated constant bias cancelled out:

\section{((HV frontal bias with right grip (R and RL)}

$-\mathrm{HV}$ frontal bias with left grip (L and LR)) /2)

HV uncertainty measures were defined in both sagittal and frontal planes (vi/vii) corresponding to intra-individual standard deviation across trials (HV sagittal uncertainty; HV frontal uncertainty).

\section{Statistical methods}

For statistical tests we reported a two-sided $P$-value $(\alpha$ level: $P<0.05)$. For background measures, a one-way ANOVA was used to compare age between groups. Welch's ANOVA and Games-Howell post hoc test were used to compare vibration thresholds between groups owing to heteroscedasticity. For experimental measures we adopted a uniform approach using mixed-effects linear regression model procedures in SPSS (IBM SPSS Statistics 26, IBM Corp., Armonk, NY, USA). In short, for all analyses, models included fixed effects for group, allowing for different variances in the three groups. Repeated measures analyses including group in addition to experimental factors (VVframe bias and VVframe uncertainty; all haptic-vertical measures) included random effects for participant to allow for clustered data.

Group and experimental factors: for visual-vertical and mechanistic model analyses, fixed effects were limited to group with the exception of VVframe bias and uncertainty analyses: these included two direction-specific means or SDs per participant and fixed effects of group and tilt (left, right) plus their interaction. All haptic-vertical analyses included a total of four grip and side-specific means or SDs per participant and the following fixed effects: group, grip (unimanual, bimanual), side (left, right). The exception was HVside-directional bias, which only included two grip-specific means per participant and correspondingly included effects of grip, but not side. For HV frontal bias, two two-way interactions were included between group and side and grip and side. For all analyses, interaction terms were included only if they significantly improved model fit based on information criteria. A global test assessed evidence for a difference across the three groups; pairwise comparisons were only implemented if evidence of an overall difference was found. To assess the proportion of participants with abnormal model estimates, given the restricted control sample $(n<50)$ a $t$-distribution was used to estimate the reference range within which $95 \%$ of the control group's mechanistic model estimates fell (Crawford \& Garthwaite, 2002; Crawford \& Howell, 2010).

For visual-vertical and haptic-vertical analyses, the effect of age was considered owing to evidence of overall and pairwise differences in age (see 'Participants' section and Table 1). However, when including age as a covariate there was no evidence of an effect of age on any of the experimental measures and there was no significant improvement in model fit based on information criteria or likelihood ratio test results. Therefore, reported results are not adjusted for age.

\section{Brain image acquisition and analysis}

T1-weighted volumetric magnetic resonance (MR) scans were acquired on a Siemens Prisma 3T scanner using a Magnetisation Prepared Rapid Acquisition Gradient Echo sequence (Echo time $(\mathrm{TE})=4.0 \mathrm{~ms}$, Repetition time $(\mathrm{TR})=8.6 \mathrm{~ms}$, Time inversion $(\mathrm{TI})=900 \mathrm{~ms}$ ) for a subset of patient participants (14 PCA; 15 tAD). Images were obtained with $282 \mathrm{~mm}$ field of view and $256 \times 256$ acquisition matrix.

Voxel-based morphometry (VBM) was performed using SPM12.1, and executed in MATLAB 2014b (The MathWorks Inc., Natick, MA, USA). Image pre-processing involved the following steps: image conversion from DICOM image format to NIFTI format, simultaneous tissue segmentation and bias correction using the Unified Segmentation algorithm (Ashburner \& Friston, 2005), the creation of a study-specific grey matter (GM) segment template using geodesic shooting (Ashburner \& Friston, 2011), normalisation of the segments to standard space (Montreal Neurological Institute (MNI) template) using the resulting transformations. Warped GM maps were modulated to preserve tissue volume and smoothed using a Gaussian kernel with $6 \mathrm{~mm}$ full width at half-maximum (FWHM) before analysis. A whole-brain grey matter mask was defined to include voxels for which the intensity was $>0.1$ in at least $90 \%$ of the images; this has been shown to be appropriate for participants with greater atrophy (Ridgway et al. 2009).

SnPM, a SPM toolbox (http://www.nisox.org/Software/ SnPM13/), yields pseudo $t$-statistic images which are then assessed for significance using a standard non-parametric multiple comparisons procedure based on permutation testing. Grey matter volume between patients was compared using SnPM's '2 Group: Two Sample $t$ test' 
Table 2. Estimated mean increases in perceptual bias and uncertainty between PCA, Control and tAD groups in the frontal plane

\begin{tabular}{|c|c|c|c|c|c|c|}
\hline & \multicolumn{3}{|c|}{ A. Increased bias $(95 \% \mathrm{Cl})\left({ }^{\circ}\right)$} & \multicolumn{3}{|c|}{ B. Increased perceptual uncertainty $(95 \% \mathrm{Cl})\left(^{\circ}\right)$} \\
\hline & PCA vs. Con & tAD vs. Con & PCA vs. tAD & PCA vs. Con & tAD vs. Con & PCA vs. tAD \\
\hline $\begin{array}{l}\text { (i) Visual vertical } \\
\text { without cues }\end{array}$ & $2.96(1.59,4.33)$ & $1.40(0.43,2.36)$ & $1.56(0.05,3.07)$ & $2.09(1.20,2.97)$ & $0.78(0.20,1.36)$ & $1.31(.34,2.28)$ \\
\hline $\begin{array}{l}\text { (ii) Visual vertical } \\
\text { with cues }\end{array}$ & $7.38(5.26,9.50)$ & $5.75(2.74,8.76)$ & $1.63(-1.54,4.80)$ & $2.41(1.30,3.52)$ & $0.56(0.17,0.96)$ & $1.85(0.71,2.99)$ \\
\hline (iii) Haptic vertical & $9.29(6.69,11.88)$ & $3.02(0.66,5.38)$ & $6.27(3.60,8.94)$ & $1.24(0.80,1.69)$ & $0.33(-0.02,0.68)$ & $0.91(0.43,1.39)$ \\
\hline
\end{tabular}

A, estimated increases in subjective VV bias without (i, VV magnitude bias) and with tilted frame cues (ii, VVframe-directional bias) and subjective HV bias (iii, HVside-directional bias). B, estimated increases in (i) subjective VV perceptual uncertainty without and (ii) with tilted frame cues, and (iii) subjective HV perceptual uncertainty. Overall estimates are averaged over grip (Aiii), tilt (Bii), and grip and side conditions (Biii).

plugin module. Prior to analysis, voxel variance estimates were pooled over neighbouring voxels in each scan using SnPM's variance smoothing feature using a $6 \mathrm{~mm}$ FWHM Gaussian kernel. Statistical significance was determined by permutation testing $(30,000$ permutations) based on peak-voxel inference and set at $P<0.05$ (family-wise error corrected, FWE), with visualisation in Fig. 1 set to $P<0.001$ (uncorrected) to allow a better characterisation of the potential differences between the groups.

\section{Results}

Table 2 shows estimated mean increases in subjective visual and haptic vertical constant bias and uncertainty between PCA, tAD and control groups in the frontal plane.

\section{Perceptual bias}

Subjective visual vertical without cues. Trials without visual orientation cues (Fig. $2 A$ grey circle background) yielded raw VV estimates in the frontal plane. Bias direction did not differ between groups overall as participants' biases were spread evenly between positive and negative values leading to group means close to zero (VV bias estimated mean increase (95\% CI); PCA vs. Controls: $0.05^{\circ}(-2.31,2.42)$; PCA vs. tAD: $0.55^{\circ}$ $(-2.07,3.16)$; tAD vs. Controls: $-0.49^{\circ}(-2.08,1.09)$; global test: $P=0.811)$. However, $\mathrm{VV}$ magnitude bias differed significantly between groups overall $(P<0.0001$; Table 2Ai), being greatest in PCA followed by tAD and control groups (Fig. 3A).

Subjective visual vertical with tilted frame cues. Trials with visual orientation cues (Fig. $2 A$ grey square background tilted $18^{\circ}$ clockwise or counterclockwise) reflected the effect of a tilted frame on subjective visual vertical. Across all groups, the tilted square background had a profound effect on VVframe bias, deviating approximately symmetrically in the direction of frame tilt (mean (95\% CI): left tilted frame, $6.45^{\circ}$ (5.11, 7.79); right tilted frame, $\left.-6.98^{\circ}(-8.31,-5.64)\right)$. This effect of frame tilt differed between groups overall (group $\times$ tilt direction: $P<0.0001$ ). Relative to controls, frame tilt had a greater effect on directional bias in either direction in PCA $(P<0.001)$ and tAD groups $(P<0.001$; Fig. $3 C$ and $E)$, though there was no evidence that the effect of tilt differed between patient groups $(P=0.22)$. VVframe-directional bias was determined by averaging signed bias relative to frame tilt across both tilt conditions, equivalent to frame-induced VV bias. Consistent with VVframe bias, VVframe-directional bias differed between groups overall $(P<0.0001)$, with evidence of increases in either patient relative to control groups and no evidence of differences between patient groups (Table 2Aii).

Subjective haptic vertical. With eyes closed, participants grasped the bottom half of the rod with their left or right hand using either a unimanual grip (L, R) or a bimanual grip with the opposite hand grasping the top half of the rod (LR, RL; Fig. 2B). Consistent directional biases were observed across all groups that depended on both these factors of side and grip. HV directional bias was greater for unimanual than bimanual grips regardless of group or measurement plane (Fig. $4 A, B, D$ and $E ; P<0.0001$ ). Crucially, the direction of the bias depended on grip side and measurement plane.

In the frontal plane (HV frontal bias; Fig. $4 A$ and $B$ ), bias direction was highly dependent on side $(P<0.0001)$. Across groups, the bias showed a positive angle when the right hand held the bottom of the stick (combining $\mathrm{R}$ and RL grips, mean $\left.(95 \% \mathrm{CI}): 6.33^{\circ}(5.26,7.39)\right)$ and a negative angle with the left hand (combining $\mathrm{L}$ and LR grips, $\left.-6.50^{\circ}(-7.56,-5.44)\right)$. This side effect differed between groups overall (group $\times$ side: $P<0.0001$ ), being greatest in the PCA followed by tAD and control groups (all $P<0.001$ ). HVside-directional bias was determined by averaging signed bias in the frontal plane relative to 
the hand holding the bottom of the stick across both side conditions. HVside-directional bias was equivalent to side-induced $\mathrm{HV}$ bias, analogous to frame-induced VV bias (VVframe-directional bias). Consistent with directional bias, across grips, HVside-directional bias was largest in PCA (mean (95\% CI): $11.60^{\circ}$ (9.62, 13.58)) followed by $\operatorname{tAD}\left(5.33^{\circ}(3.45,7.21)\right)$ and control groups $\left(2.31^{\circ}(0.55,4.07)\right.$; global test: $\left.P<0.001\right)$. Pairwise comparisons showed that all three groups differed significantly from each other (Table 2Aiii). As with HV directional bias, HVside-directional bias was greater for unimanual than bimanual grips across groups (unimanual: $8.95^{\circ}(7.81,10.09)$; bimanual: $3.87^{\circ}(2.73$, 5.02); $P<0.001)$.
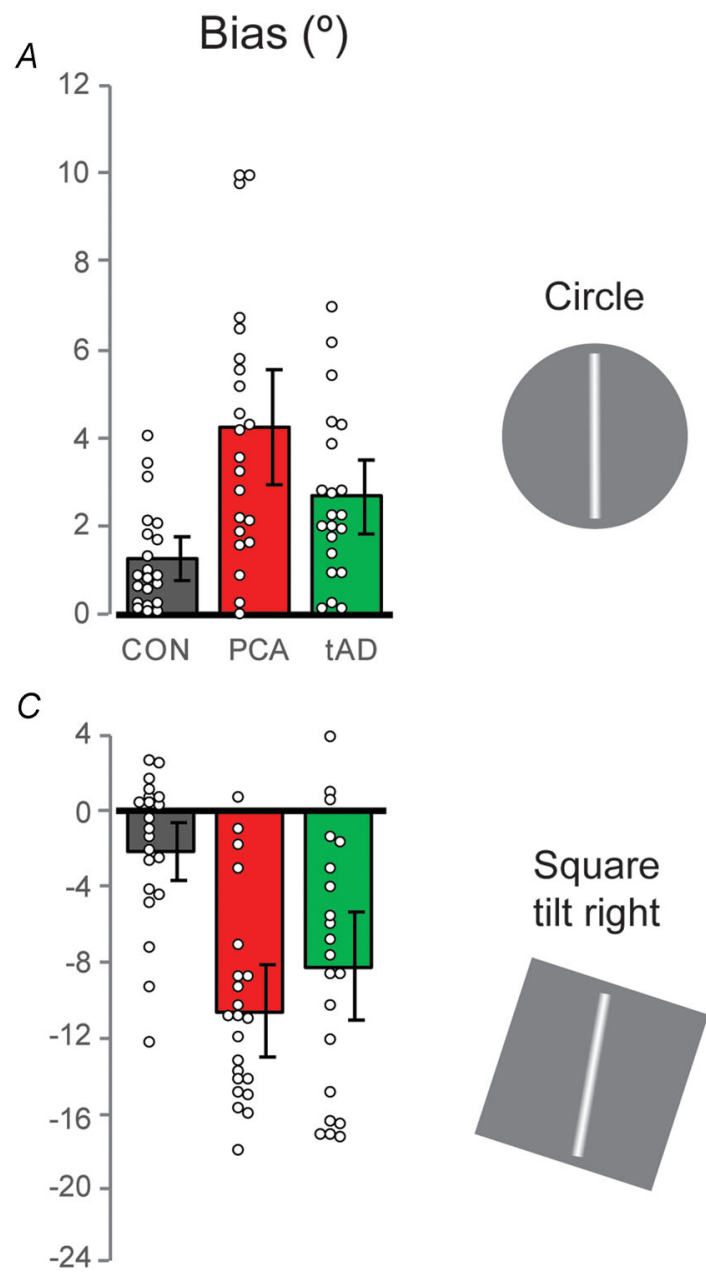

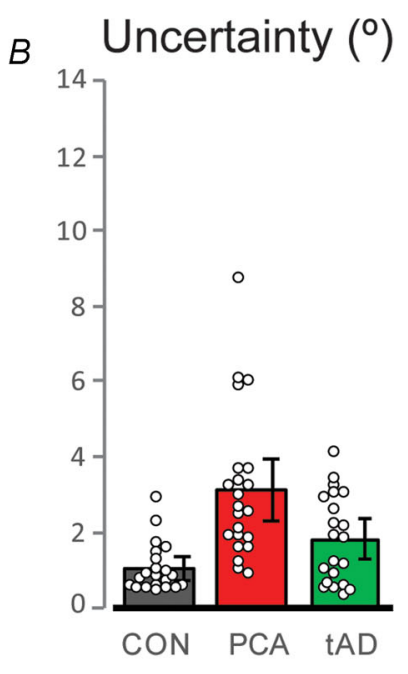

$D$

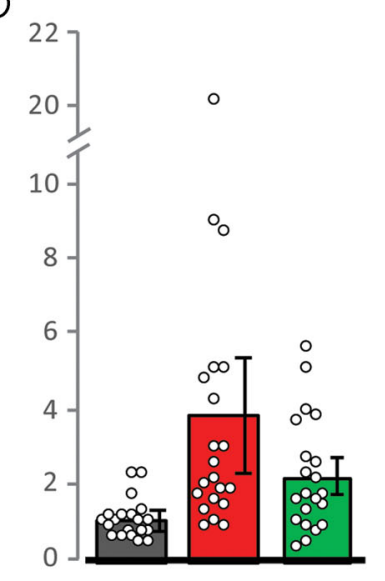

F

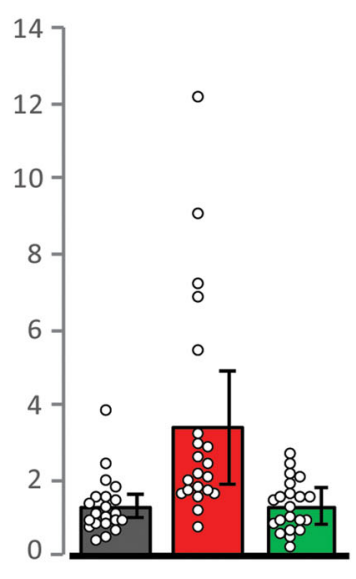

Figure 3. Visual-vertical rod angle bias and uncertainty

Circles show observed individual participant values; coloured bars represent estimated group means and error bars correspond to 95\% Cls. Bias with background grey circle ( $A, \mathrm{~V}$ magnitude bias) is calculated from participant absolute mean rod angle. Bias with background grey tilted square ( $C$ and $E, V V$ frame bias) is calculated from participant signed mean rod angle. Uncertainty $(B, W V$ uncertainty; $D$ and $F$, VVframe uncertainty; right panels) is calculated from participant intra-individual standard deviation rod angle. Colour code: grey, controls; red, posterior cortical atrophy; green, typical Alzheimer's disease. 
In the sagittal plane, there was an overall bias in the positive (forward) direction (HV sagittal bias; Fig. 4D and $E$ ), but no evidence this bias differed between group (HV sagittal bias estimated mean increase (95\% CI); PCA vs. Controls: $1.47^{\circ}(-1.04,3.98)$; PCA vs. tAD: $1.38^{\circ}$ $(-1.21,3.98)$; tAD vs. Controls: $0.09^{\circ}(-2.25,2.44)$; global test: $P=0.457)$. In contrast to HV frontal bias, there was no effect of side on HV sagittal bias (combining R and RL grips, mean (95\% CI): $4.53^{\circ}(3.39,5.66)$; combining $\mathrm{L}$ and LR grips, $\left.4.59^{\circ}(3.46,5.73) ; P=0.901\right)$.

\section{Perceptual uncertainty}

Perceptual uncertainty associated with verticality estimates consistently differed between groups for both visual (Fig. 3B, $D$ and $F$ ) and haptic (Fig. $4 C$ and $F$ ) tasks, and it was always greatest in PCA followed by tAD and control groups. Pairwise comparisons consistently showed that PCA differed significantly from both tAD and control groups (Table 2B); tAD tended to have greater uncertainty than the control group with the exception
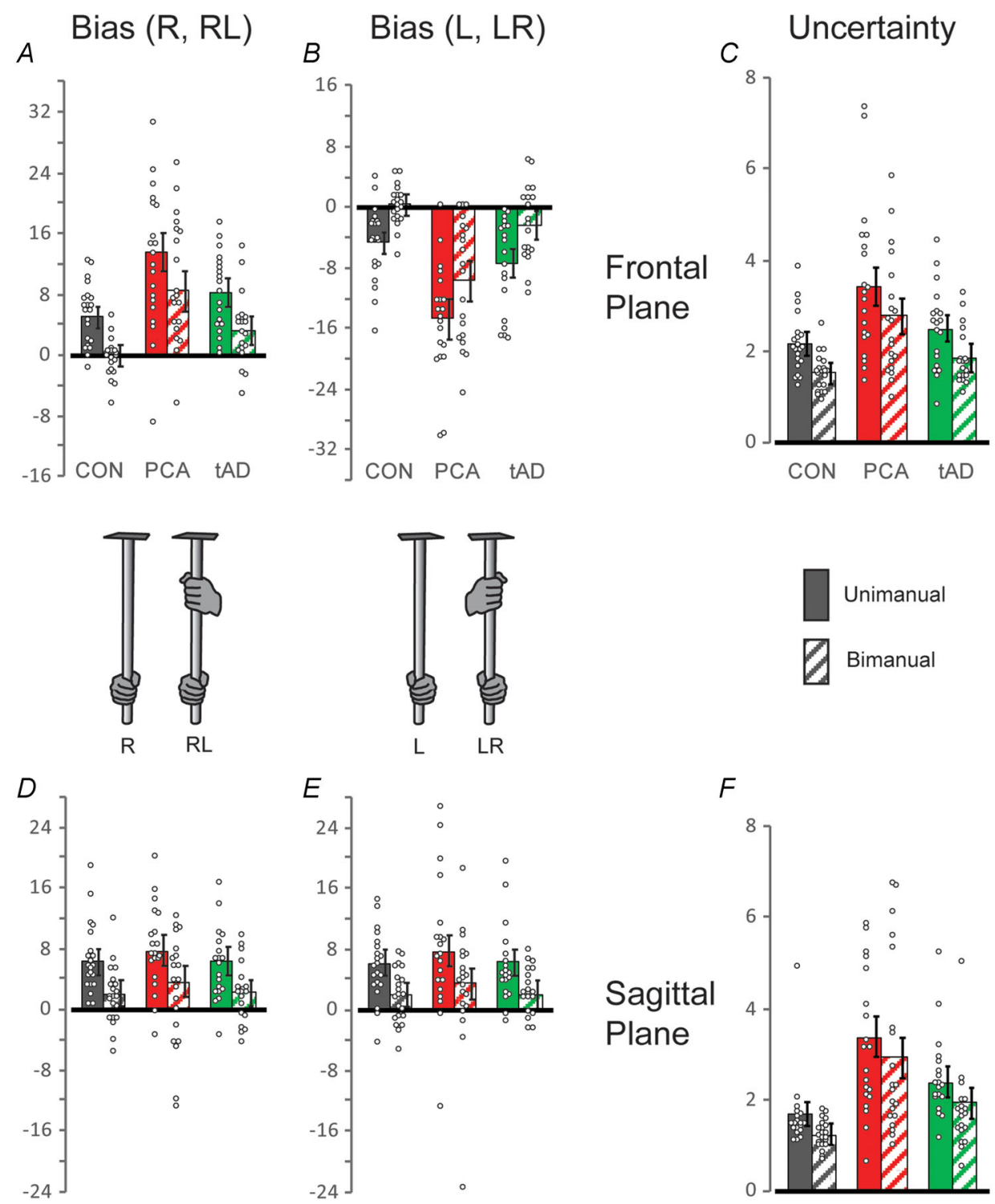

Figure 4. Haptic-vertical rod angle bias and uncertainty

Circles show observed individual participant values; coloured bars represent estimated group means and error bars correspond to $95 \% \mathrm{Cls}$. Bias calculated from participant signed mean rod angle in the frontal plane ( $A$ and $B, \mathrm{HV}$ frontal bias) and sagittal plane ( $D$ and $E, H V$ sagittal bias), shown separately from right hand $(A$ and $D)$; and left hand grips ( $B$ and $E$ ). Uncertainty ( $C, H V$ frontal uncertainty; $F, H V$ sagittal uncertainty) is calculated from participant intra-individual standard deviation rod angles combined across grip conditions. Unimanual grips (filled colours) are distinguished from bimanual grips (hatched colours). Colour code: grey, controls; red, posterior cortical atrophy; green, typical Alzheimer's disease. 
of $\mathrm{HV}$ in the frontal plane where the difference was not formally statistically significant. For the haptic task, the uncertainty associated with unimanual grip was always significantly greater than for bimanual grip across all groups.

\section{Mechanistic model}

Given the multisensory nature of tasks, a model was developed which considered participants' own estimates of subjective visual- and haptic-vertical as the product of multiple mechanisms. The model was based on principles of maximum likelihood estimation to estimate the uncertainty associated with a candidate primary mechanism (Abstract Figure). In short, each participant's subjective verticality estimate was defined by their observed perceptual bias and uncertainty when repeatedly orientating a rod to Earth vertical under different task conditions (visual-vertical, Fig. 3; haptic-vertical, Fig. 4). Subjective verticality estimates were assumed to result from independent estimates associated with primary and secondary mechanisms, combined in a statistically optimal way. Primary and secondary mechanism estimates were considered likely to differ and each have their own and different degree of uncertainty. By weighting each according to its degree of uncertainty and combining them, a subjective verticality estimate was obtained that has an intermediate mean value and a degree of uncertainty less than that arising from either of the primary or secondary mechanisms. While primary and secondary mechanism estimates are unobserved, primary mechanism uncertainty may be estimated using observed bias and uncertainty measures and assumptions particularly regarding secondary mechanisms, outlined below.

In detail, participants' subjective vertical estimates (SV, Abstract Figure) were assumed to be normally distributed with a mean $\mu$ and standard deviation $\sigma$ corresponding to observed bias and uncertainty, and to result from the product of underlying estimates (primary: I; secondary: II) which are themselves normally distributed. The three Gaussians are related by the following:

$$
\begin{gathered}
\mu_{\mathrm{SV}}=\frac{\left(\mu_{\mathrm{I}} \sigma_{\mathrm{II}}^{2}+\mu_{\mathrm{II}} \sigma_{\mathrm{I}}^{2}\right)}{\sigma_{\mathrm{I}}^{2}+\sigma_{\mathrm{II}}^{2}} \\
\frac{1}{\sigma_{\mathrm{sv}}^{2}}=\frac{1}{\sigma_{\mathrm{I}}^{2}}+\frac{1}{\sigma_{\mathrm{II}}^{2}}
\end{gathered}
$$

Visual-vertical primary and secondary mechanisms. In the absence of any verticality cues (grey circle background), observed visual-vertical (VV1) parameters $\left(\mu_{\mathrm{VV} 1} \sigma_{\mathrm{VV} 1}\right)$ are modelled as the product of a secondary mechanism (V1), arising from a retinal representation of $\operatorname{vertical}\left(\mu_{\mathrm{V} 1} \sigma_{\mathrm{V} 1}\right)$, and a primary mechanism (G1), arising from a graviceptive representation of vertical $\left(\mu_{\mathrm{G} 1}, \sigma_{\mathrm{G} 1}\right)$. An example of this is illustrated in Fig. $5 A$ in which any underlying bias of each estimate has been removed. The standard deviation of this combined estimate represents the uncertainty of the observed visual-vertical obtained under these restricted visual conditions.

When the tilted frame is present, the independent sources of verticality are now threefold. They consist of the primary mechanism (G1) and two secondary mechanisms arising from the principal retinal axis reference (V1) and a square-image (frame) reference (V2). As illustrated in Fig. $5 B$, these three sources are combined to yield the observed visual-vertical parameters (VV2) when the rod is superimposed on the tilted square.

In reality, we do not have access to these underlying values (G1, V1, V2) for each individual. We have only the observed values of the means and standard deviations of each participant's visual-vertical attempts without and with the influence of the tilted frame (VV1 VV2). The new visual-vertical $\left(\mu_{\mathrm{VV} 2}, \sigma_{\mathrm{VV} 2}\right)$ is therefore modelled as the product of the additional retinal cue afforded by the frame $\left(\mu_{\mathrm{V} 2}, \sigma_{\mathrm{V} 2}\right)$ and the visual-vertical without it $\left(\mu_{\mathrm{VV} 1} \sigma_{\mathrm{VV} 1}\right)$. If we define $\mu_{\mathrm{V} 2}$ as the observed frame effect, which is the effect relative to any underlying bias, we can let $\mu_{\mathrm{V} 1}=$ $\mu_{\mathrm{G} 1}=0$, and as the frame was tilted by $18^{\circ}, \mu_{\mathrm{V} 2}=18$. Substituting into eqns (1) and (2) it can be shown that:

$$
\sigma_{\mathrm{G} 1}^{2}=\frac{\sigma_{\mathrm{VV} 1}^{2}}{1-\frac{\left(\mu_{\mathrm{VV} 2} \sigma_{\mathrm{VV} 1}^{2}\right)}{\left(18 k^{2} \sigma_{\mathrm{VV} 2}^{2}\right)}}
$$

Where

$$
k=\frac{\sigma_{\mathrm{V} 1}}{\sigma_{\mathrm{V} 2}}
$$

Primary mechanism estimates as a function of visual-vertical secondary mechanisms. Model estimates are presented from three theoretical participants given the group mean biases and standard deviations from the visual-vertical tasks without and with visual cues (Fig. 5C). Figure $5 C$ shows estimated standard deviation of the primary mechanism of subjective visual-vertical (G1) as a function of the ratio $k$, the ratio of uncertainties of the two secondary mechanism references (V1: retinal axis; V2: frame reference). Across control and patient groups, G1 standard deviation estimates become smaller as the ratio of the two uncertainties between retinal references (retinal vertical axis uncertainty divided by retinal frame uncertainty) increases, approaching a minimum value at $k=10$.

Haptic-vertical primary and secondary mechanisms. As illustrated by example in Fig. 5E, the observed haptic-vertical (HV1) with the unimanual grip $\left(\mu_{\mathrm{HV} 1}, \sigma_{\mathrm{HV} 1}\right)$ is modelled as the product of a secondary mechanism (H1), arising from a 'local' 


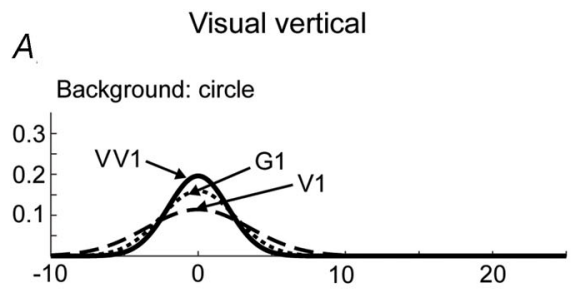

$B$

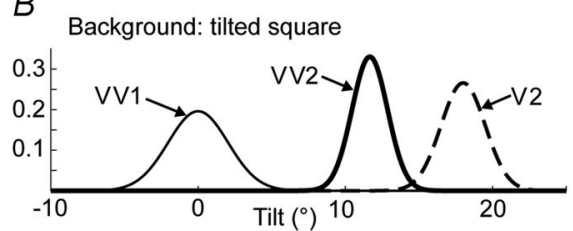

C

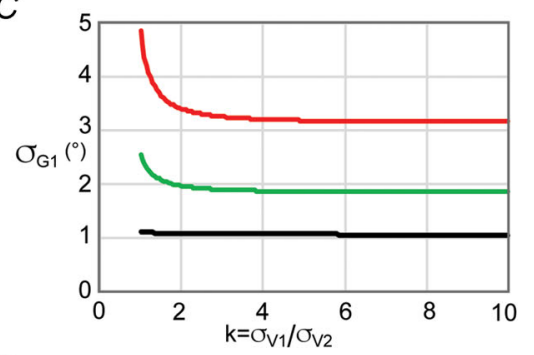

$D$

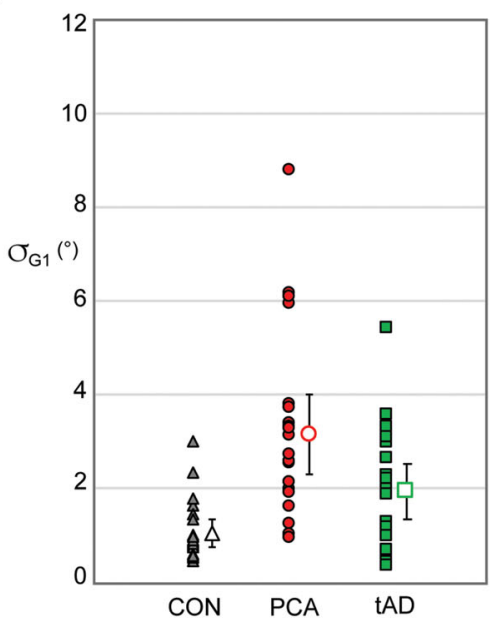

E

Haptic vertical

Grip: unimanual

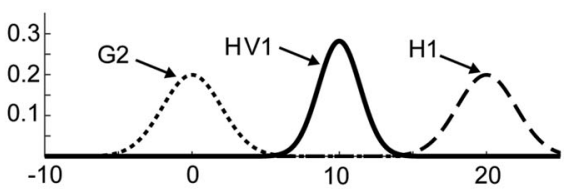

F

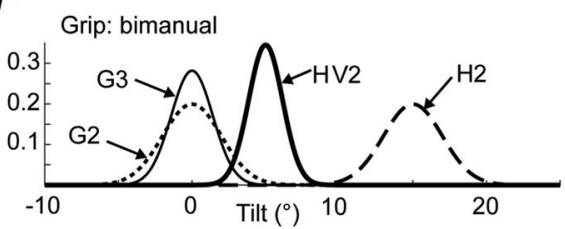

G

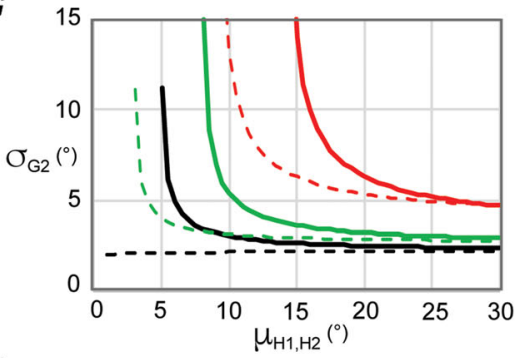

$H$

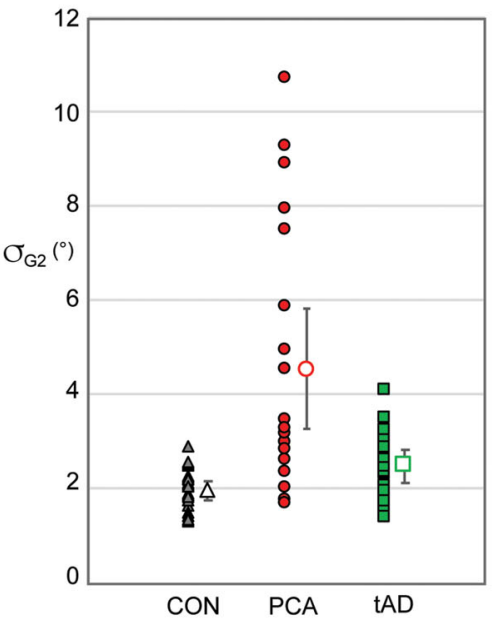

Figure 5. Mechanistic model and its predictions

From top, $A, B, E$ and $F$ show examples of probabilistic component mechanisms underlying subjective verticality estimation under the different experimental conditions represented by Gaussian curves. While model estimates incorporate observed measures of bias and uncertainty (VV1/2: visual-vertical without/with frame; HV1/2: haptic-vertical unimanual/bimanual), Gaussian curves are for illustrative purposes and do not necessarily represent any one individual. See 'Mechanistic model' section and 'Discussion' for meaning of symbols. C shows the modelled standard deviation of the estimate from the primary mechanism of the subjective visual-vertical (G1) as a function of the ratio $k$, the ratio of uncertainties of the two secondary mechanism references (V1: retinal axis; V2: frame reference). The curves are from three theoretical participants given the group mean biases and standard deviations from the two visual-vertical tasks (CON: black; PCA: red; tAD: green). G shows the modelled standard deviation of the estimate from the primary mechanism of the subjective haptic-vertical (G2) as a function of the bias of the secondary mechanism ( $\mathrm{H} 1$ : unimanual; $\mathrm{H} 2$ : bimanual). The curves are shown separately for the unimanual (continuous line) and bimanual (dashed line) tasks from three theoretical participants given the group mean biases and standard deviations from the two haptic-vertical tasks (colour coding as C). D, modelled standard deviation of the visual primary mechanisms (with $k=10$ ) for each participant (filled symbols) and each group's estimated marginal means (open symbols) and their $95 \% \mathrm{Cl}$. $\mathrm{H}$ modelled standard deviation of the haptic primary mechanism (average of unimanual and bimanual uncertainty values in the frontal plane with respective $\mu_{\mathrm{H}}=30^{\circ}$ ) for each participant (filled symbols) and each group's estimated marginal means (open symbols) and their 95\% Cl. 
hand-based representation of vertical $\left(\mu_{\mathrm{H} 1}, \sigma_{\mathrm{H} 1}\right)$, and a primary mechanism (G2), arising from a graviceptive representation of vertical $\left(\mu_{\mathrm{G} 2}, \sigma_{\mathrm{G} 2}\right)$. The primary mechanism (G2) underlying the unimanual haptic-vertical is allowed to differ from its visual-vertical counterpart (G1) since the spatial transforms from graviceptors to eye and hand differ. If we let $\mu_{\mathrm{G} 2}=0$, by substituting into eqns (1) and (2) we obtain

$$
\sigma_{\mathrm{G} 2}^{2}=\frac{\left(\mu_{\mathrm{H} 1} \sigma_{\mathrm{HV} 1}^{2}\right)}{\left(\mu_{\mathrm{H} 1}-\mu_{\mathrm{HV} 1}\right)}
$$

The observed haptic-vertical (HV2) using a bimanual grip $\left(\mu_{\mathrm{HV} 2}, \sigma_{\mathrm{HV} 2}\right)$ is modelled as the product of 'local' and a graviceptive representation of vertical, similar to the unimanual model. We do not assume that the bias and uncertainty associated with the secondary mechanism for the bimanual grip (H2) is the same as for the unimanual grip (H1). We do, however, assume that the reliability of the primary mechanism is related for the two grips. Specifically, we model the bimanual primary mechanism (G3) as the product of two unimanual representations (G2), one for each hand. From eqn (2) this gives a bimanual graviceptive variance that is half the unimanual graviceptive variance, as illustrated by an example in Fig. $5 F$. It can then be shown that

$$
\sigma_{\mathrm{G} 2}^{2}=\frac{2\left(\mu_{\mathrm{H} 2} \sigma_{\mathrm{HV} 2}^{2}\right)}{\left(\mu_{\mathrm{H} 2}-\mu_{\mathrm{HV} 2}\right)}
$$

Primary mechanism estimates as a function of haptic-vertical secondary mechanisms. Model estimates are presented from three theoretical participants given the group mean biases and standard deviations from the haptic vertical unimanual and bimanual tasks (Fig. $5 G$ ). Figure $5 G$ shows estimated standard deviation of the primary mechanism of subjective haptic-vertical (G2) as a function of the bias of the secondary mechanism (H1: unimanual; H2: bimanual). For the haptic task, G2 standard deviation estimates become smaller as haptic biases increase, approaching minimum values at $\mu_{\mathrm{H} 1, \mathrm{H} 2}=30^{\circ}$.

\section{Estimated primary mechanism uncertainty in PCA, tAD and Controls}

While key parameters of interest (V1, V2, H1, H2) are unknown, primary mechanism standard deviation (uncertainty) values approaching conservative minima may be estimated by specifying secondary mechanism values (see above, Fig. 5C and $G$ ). Formal analyses of these conservative estimates provided evidence that primary mechanism uncertainty was greater in both patient groups relative to controls across tasks, being greatest in PCA (Fig. $5 D$ and $H$ ).
For visual-vertical tasks, estimated mean increases in uncertainty were roughly $1-2^{\circ}$ between groups (G1 estimated mean increase (95\% CI); PCA vs. Controls: $2.11^{\circ}(1.21,3.00)$; PCA vs. tAD: $1.24^{\circ}(0.23,2.25)$; tAD vs. Controls: $0.87^{\circ}(.22,1.51)$; global test: $\left.P<0.001\right)$. G1 uncertainty was outside the reference range in 13/22 (59\%) PCA and 7/21 (33\%) tAD compared to $1 / 21$ (5\%) control participants.

For haptic-vertical tasks averaging across bimanual and unimanual conditions, estimated mean increases in uncertainty were roughly $0.5-2.5^{\circ}$ between groups, being $>2^{\circ}$ in PCA compared to both control and tAD groups (G2 estimated mean increase (95\% CI); PCA vs. Controls: $2.57^{\circ}(1.29,3.86)$; PCA vs. tAD: $2.05^{\circ}(0.74,3.36)$; tAD vs. Controls: $0.52^{\circ}(0.13,0.91)$; global test: $\left.P<0.001\right)$. G2 uncertainty was outside the reference range in $13 / 20$ (65\%) PCA and 5/19 (26\%) tAD compared to 0/21 control participants.

\section{Discussion}

We have assessed verticality perception in PCA and tAD determined by what looks (visual-vertical) or feels (haptic-vertical) upright. Disturbed verticality perception manifested as either excessive trial-to-trial variation of verticality estimates, reflecting enhanced perceptual uncertainty, or exaggerated constant directional bias in their estimates. Both PCA and tAD groups showed evidence of disturbed perception relative to healthy controls. With the exception of visual-vertical performance with visual cues, there was evidence of increased perceptual bias and uncertainty in the PCA compared with the tAD group across visual and haptic-vertical tasks, with disturbances being particularly apparent on haptic-vertical performance. This first major systematic investigation of verticality perception in PCA and $\mathrm{tAD}$ provides evidence of disturbed multisensory integration, notable also in the absence of visual information in PCA, often considered the cardinal 'visual dementia'.

The findings emphasise disturbances in spatial orientation and postural control in PCA that cannot be accounted for by deficits restricted to the visual domain. Relative to tAD and control groups, the PCA group exhibited abnormal verticality perception on the haptic vertical task in the absence of visual feedback. While the findings do not preclude visual influences on abnormal haptic vertical performance (for example, given somatosensory activity modulated by vision in healthy participants; Taylor-Clarke et al. 2002), they implicate non-visual disturbances as contributors to initial descriptions of PCA involving unreliable transference from standing to sitting, difficulty finding sleeves of clothes and unreliable determination of heading direction within the home (Benson et al. 1988; Nestor 
et al. 2003). The heightened effects of visual orientation cues in both PCA and tAD relative to control groups follows recent evidence of effects of visual contrast cues facilitating visually guided navigation in combined PCA and $\mathrm{tAD}$ groups, suggestive of the exaggerated role of the visual environment (Yong et al. 2018). Beyond promoting consideration of multisensory features in PCA and $\mathrm{tAD}$, the findings underline 'spatial' components of 'visual-spatial AD' and recommendations for appreciation of non-visual symptoms in non-pharmacological treatment of PCA (Graff-Radford et al. 2021).

To interpret the findings in more detail we discuss and explicitly model possible mechanisms that contribute to verticality perception under the current experimental conditions, particularly in the context of combining and spatially transforming sensory information. Disordered spatial transformations have received attention in the context of spatial navigation deficits in tAD (Coughlan et al. 2018), and may be a component of marked deficits in visually guided navigation in PCA and tAD (Yong et al. 2018).

\section{The primary mechanism}

The primary source of sensory information indicating verticality comes from sensory receptors responding to gravitational force (graviceptors). Strictly, graviceptors provide an ambiguous signal of gravity since in general they respond to any linear acceleration of the body, not just from gravity's equivalent acceleration (Angelaki et al. 1998). However, for conditions including the current investigations where the participant is essentially at rest, graviceptors reliably transduce the forces arising from gravity. One of the main processing tasks the brain must perform on graviceptive signals is to transform them between spatial coordinate systems; the directional signal emanating from a graviceptive system only reports the direction of gravity in the coordinate frame of the body part in which the graviceptors are embedded. For example, the vestibular otoliths embedded in the skull signal gravity direction in a head-fixed reference frame. Orienting a hand-held rod to vertical based on this information should therefore require additional information about the hand's orientation relative to the skull. Thus, a head-fixed to hand-fixed coordinate transformation would be needed, which requires information about the orientation of each body part between the hand and the head (Barra et al. 2010). Similar considerations apply to other body-embedded graviceptors; the kidneys and large blood vessels can act as inertial sensors and provide gravity information (Mittelstaedt, 1996), as can mechano-receptors in the skin that contact external surfaces (such as the floor or a chair) to support body weight (Lackner \& Dizio, 2005). We shall refer to the totality of these weighted systems of graviceptors and their respective spatial transformations as the primary mechanism.

\section{Disturbance of visual-vertical}

With the rod superimposed on a featureless grey circle, both PCA and tAD groups performed worse than controls, with PCA performing worse than $\mathrm{ADD}$. Poor performance manifested as a larger absolute bias (PCA 335\% and tAD $211 \%$ relative to controls), but with no overall directional tendency within a group, and greater intra-individual variability (PCA 296\% and tAD 174\% relative to controls). Under this condition, the primary mechanism arguably provides the main reference of verticality. However, with the participant seated and head held in an upright position, an approximate task solution might additionally be provided by aligning the rod to a retinal axis that has been associated with vertical images through experience. This would constitute a 'local' secondary mechanism for estimating verticality and unlike the primary mechanism does not require spatial transformation. The observed visual-vertical biases and uncertainties can thus be considered the final estimate of verticality after estimates from primary and secondary mechanisms have been combined.

The hypothesised secondary mechanism may be a significant source of variability for the PCA group as it relies on accurate processing of retinal information, and visual processing deficits are typically considered the defining feature of PCA (see Table 1). However, observations from the tilted-frame test provide evidence for a disturbance of the primary mechanism. With the rod superimposed on a tilted grey square background, the estimate of verticality became biased in the direction of frame tilt. While this is a normal, well-documented and robust phenomenon in healthy participants (Vingerhoets et al. 2009), both patient groups were excessively and similarly biased by the tilted frame. Relative to controls, mean rod tilt with frame was $415 \%$ and $346 \%$ greater in PCA and tAD groups, respectively. The effect was substantial and for some patients the rod could be tilted by more than $50 \%$ of the $18^{\circ}$ tilted square background (group mean tilt: PCA $9.7^{\circ}$; tAD $8.1^{\circ}$ ). The frame effect can be explained by the retinal image of the square providing a reference for an additional 'local' secondary mechanism. The retinal image of the rod can now be aligned to two possible local references, one defined by the principal axis of the eye and the other by the square.

As shown in the derivation of the mechanistic model, with some assumptions and with observed visual-vertical uncertainty and bias we can deduce the degree of uncertainty of an individual's primary mechanism (G1). Figure $5 C$ shows the result of this calculation for three theoretically plausible individuals assigned mean values of visual-vertical biases and uncertainties from each of the 
three groups. The estimated uncertainty of the primary mechanism is a function of the ratio of uncertainties associated with the two retinal references within the secondary mechanism (Fig. 5C). The estimated value of the primary mechanism uncertainty is greatest when the ratio approaches unity ( $k=1$, i.e. identical uncertainties for the two retinal references). This then decreases towards an asymptotic value as the ratio increases, that is, less uncertainty associated with the tilted square retinal reference, which is likely given that orientations of the rod and square can be directly compared whereas the orientation of a retinal axis based on association is relatively nebulous.

\section{Disturbance of haptic-vertical (absence of vision)}

The consistent side-specific haptic-vertical biases observed in the frontal plane in all three groups would be difficult to explain if verticality estimation was predicated solely on the primary mechanism. When held in the right hand, the top of the rod was tilted towards the participant's left, and vice versa when held in the left hand. Even when the opposite hand grasped the top of the rod in a bimanual grip, the bias remained but with reduced magnitude. We therefore suggest that estimates of haptic vertical are influenced by a local secondary mechanism analogous to those involved in visual-vertical estimates.

One candidate basis of a secondary mechanism is the gravitational force acting on the rod allowing verticality estimation. This gravitational force would be resisted by forces between rod and hand, sensed by skin mechanoreceptors. The resistive force will comprise a shear force component parallel to the rod's long axis and an orthogonal normal force component; while the latter will also reflect grip pressure, this will have no net resultant. If held vertically, the only force from the rod's weight will be the shear force; correspondingly, reducing the net orthogonal normal force to zero offers a task solution without requiring spatial transformation. A source of the observed side-specific biases may relate to the manner of contact between hand and rod. The palm predominantly contacts the lateral aspect of the held rod, with digits additionally contacting medial aspects. Non-uniform distribution of palm and digit mechanoreceptors implies non-uniform haptic acuity of rod normal forces around the circumference of the rod. A directional bias may arise from constant errors in haptic estimates of net normal force corresponding to zero, that is, indicating veridical vertical. While other secondary mechanisms are possible, this proposed secondary mechanism may explain observed asymmetrical biases being mirror-reversed in the frontal plane when the rod was held in right $v s$. left hand (leftward vs. rightward tilt), while being consistent in sagittal plane (forward tilt; Fig. $4 A, B, D$ and $E$ ).
Regardless of its nature, the effect of a biased secondary mechanism on verticality perception would be tempered by a presumed unbiased, but uncertain, primary mechanism. Both patient groups produced excessive side-related tilts of the rod in the frontal plane compared with controls, although the PCA group was by far the greater affected of the two (PCA 502\% and tAD 231\% relative to controls). The explanation we favour is that the primary mechanism is less reliable in the patient groups and thus less effective in modifying the bias introduced by the secondary mechanism.

In the mechanistic model, the uncertainty of the haptic primary mechanism is a function of the observed haptic-vertical uncertainty and bias (measured for unimanual and bimanual grips separately; HV1 and HV2) and the bias of the secondary mechanism, which has an unknown value. Figure $5 G$ plots the estimated primary-mechanism uncertainty (intra-individual standard deviation) against values of this unknown bias for three theoretically plausible individuals assigned the haptic-vertical mean values from each of the three groups. These curves show there is a minimum value of secondary-mechanism bias with which the equation can be solved. At this value, the estimate of the primary mechanism's uncertainty is at its highest. The uncertainty value rapidly reduces as the bias is increased, tending towards a stable asymptotic value. Secondly, the secondary-mechanism biases differ for unimanual and bimanual grips. In Fig. $5 G$ the two hypothetical patient examples suggest that the bimanual curve is shifted leftwards relative to the unimanual curve, indicating that in these examples the bimanual bias is of the order of $5^{\circ}$ less than the unimanual bias. This corresponds with the reasonable expectation that the net bias of a local secondary mechanism arising from one hand would be reduced when both hands are involved.

\section{Degradation of the primary mechanism in PCA vs. TAD}

The currently unknown values of key parameters in the model prohibit a definitive value of primary-mechanism uncertainty for each participant. For the visual-vertical the key unknown is $k$, the ratio of uncertainties of the two secondary mechanism references (retinal axis vs. frame reference; Fig. 5C); for the haptic-vertical the unknown is the bias of the secondary mechanism (local hand-based bias; Fig. 5G). Nonetheless, we can estimate a conservative value for each participant. As shown in the curves of Fig. $5 C$ and $G$, values of uncertainty tend towards lower values for higher levels of $k$ and bias for visual and haptic modalities, respectively. Therefore, by setting these values arbitrarily high, yet plausible $\left(k=10\right.$, bias $\left.=30^{\circ}\right)$, we obtain conservative estimates of primary-mechanism uncertainty that approach the minimum possible for each participant. 
Conservative estimates of primary-mechanism uncertainty are higher for both patient groups compared with controls (Fig. $5 D$ and $H$ ), and in PCA relative to tAD. Predominant posterior neurodegeneration may cause greater uncertainty of primary-mechanism estimates, prompting increased reliance on secondary mechanisms in estimating verticality (e.g. exaggerated frame-induced bias). Discrepancies in primary-mechanism uncertainty between PCA and tAD groups are particularly apparent based on haptic-vertical measures, possibly reflecting differences in computational demands. For the visual modality, at its simplest by assuming vestibular otoliths provide the main graviceptive input, the computation involves only a spatial transform from head to eye-centred frames. For the haptic modality, the computation involves transformation across the multiple coordinate frames of all body segments between the graviceptor host and effector; from head- and body-centred coordinates, to postural arm configuration, to the hand. Such increases in computational demands would make the process more susceptible to degradation by disease, and/or visual and haptic computations may differ in susceptibility to regional atrophy. Findings consistent with spatial transformation and multisensory deficits likely relate to core PCA clinico-radiological features, particularly posterior parietal atrophy, space misperception, optic ataxia (Pisella et al. 2009) and environmental disorientation.

\section{Strengths and weaknesses}

Strengths of the current investigation include patient participants well characterised in neuropsychological phenotype, two patient groups of comparable disease severity and a relatively large sample of PCA participants. However, the current sample of PCA patients was younger than both control and tAD groups, in line with PCA being younger onset than tAD (Schott et al. 2016), and thus broadly representative of the wider PCA and tAD populations. Even so, the greater deficits observed in the PCA group cannot plausibly be related to younger age; there was no evidence of an effect of age on any of the experimental measures, and including age did not improve model fit. While patients were excluded based on clinical features suggestive of underlying Lewy body pathology or corticobasal degeneration, molecular or pathological evidence of $\mathrm{AD}$ was only available in a subset of patients and so non-AD pathology cannot be definitively ruled out. Diminished fine motor control in patient groups was unlikely to explain their exaggerated constant biases, but could have contributed to increases in uncertainty measures. However, participants were allowed ample time to provide responses, allowing them to correct errors associated with their action. While in the current study head position was fixed for visual tasks using a custom brace informed by participant feedback (Crutch et al. 2018), future investigations might introduce varying head and whole body tilt conditions to evaluate proprioceptive and vestibular influences on verticality perception (Vingerhoets et al.2009; Alberts et al. 2019). Furthermore, assessment of both subjective horizontal and subjective vertical using visual and haptic tasks may promote understanding of spatial disorientation while minimising observed haptic vertical biases.

\section{Conclusions}

The findings demonstrate altered verticality perception in PCA and tAD suggesting a disturbance of the mechanism that spatially transforms graviceptive information from the coordinate frame of one body part to that of another. The greater deficit observed in PCA compared with tAD supports the hypothesis that posterior parietal cortex plays a role in this process. This provides insight into the nature and basis of spatial disorientation in PCA, and has implications for health-care professionals by prompting consideration of visual and non-visual/somatosensory disturbances undermining functional status in dementia.

\section{References}

Alberts BBGT, Selen LPJ \& Medendorp WP (2019). Age-related reweighting of visual and vestibular cues for vertical perception. J Neurophysiol 121, 1279-1288.

Alladi S, Xuereb J, Bak T, Nestor P, Knibb J, Patterson K \& Hodges JR (2007). Focal cortical presentations of Alzheimer's disease. Brain 130, 2636-2645.

Allan LM, Ballard CG, Rowan EN \& Kenny RA (2009). Incidence and prediction of falls in dementia: a prospective study in older people. PLoS One 4, e5521.

Andersen RA, Essick GK \& Siegel RM (1985). Encoding of spatial location by posterior parietal neurons. Science $\mathbf{2 3 0}$, 456-458.

Angelaki DE, Mchenry MQ, Dickman JD, Newlands SD \& Hess BJM (1998). Computation of inertial motion: neural strategies to resolve ambiguous otolith information.

Ashburner J \& Friston KJ (2005). Unified segmentation. Neuroimage 26, 839-851.

Ashburner J \& Friston KJ (2011). Diffeomorphic registration using geodesic shooting and Gauss-Newton optimisation. Neuroimage 55, 954-967.

Barra J, Marquer A, Joassin R, Reymond C, Metge L, Chauvineau V \& Pérennou D (2010). Humans use internal models to construct and update a sense of verticality. Brain 133, 3552-3563.

Benson DF, Davis RJ \& Snyder BD (1988). Posterior cortical atrophy. Arch Neurol 45, 789-793.

Buneo CA, Jarvis MR, Batista AP \& Andersen RA (2002). Direct visuomotor transformations for reaching. Nature 416, 632-636.

Coughlan G, Laczó J, Hort J \& Hornberger M (2018). Spatial navigation deficits-overlooked cognitive marker for preclinical Alzheimer disease? Nat Rev Neurol 14, 496-506. 
Crawford JR \& Garthwaite PH (2002). Investigation of the single case in neuropsychology: confidence limits on the abnormality of test scores and test score differences. Neuropsychologia 40, 1196-1208.

Crawford JR \& Howell DC (2010). The clinical neuropsychologist comparing an individual's test score against norms derived from small samples. Clin Neuropsychol 12, 482-486.

Crutch S, Herron D, Pickett J, Rosser S \& Rossor M (2020). Inspired by chance: valuing patients' informal contributions to research. BMJ 371, m4478.

Crutch SJ, Schott JM, Rabinovici GD, Murray M, Snowden JS, van der Flier WM, Dickerson BC, Vandenberghe R, Ahmed S, Bak TH, Boeve BF, Butler C, Cappa SF, Ceccaldi M, de Souza LC, Dubois B, Felician O, Galasko D, Graff-Radford J, Graff-Radford NR, Hof PR, Krolak-Salmon P, Lehmann M, Magnin E, Mendez MF, Nestor PJ, Onyike CU, Pelak VS, Pijnenburg Y, Primativo S, Rossor MN, Ryan NS, Scheltens P, Shakespeare TJ, González AS, Tang-Wai DF, Yong KXX, Carrillo M \& Fox NC (2017). Consensus classification of posterior cortical atrophy. Alzheimers Dement 13, 870-884.

Crutch SJ, Yong KXX, Peters A, Ocal D, Kaski D, Gonzalez AS, Ryan N, Ball S, Harrison CR, Murphy C, Hulme P, Phillips I, Hulme G, Brown A, Brown L, Riley P, Ramsey L, Woods A \& Day B (2018). Contributions of patient and citizen researchers to 'Am I the right way up?' study of balance in posterior cortical atrophy and typical Alzheimer's disease. Dementia 17, 1011-1022.

Dakin CJ, Peters A, Giunti P \& Day BL (2018). Cerebellar degeneration increases visual influence on dynamic estimates of verticality. Curr Biol 28, 3589-3598.e3.

Dakin CJ \& Rosenberg A (2018). Gravity Estimation and Verticality Perception, 1st edn. Elsevier B.V.

Duhamel JR, Colby CL \& Goldberg ME (1998). Ventral intraparietal area of the macaque: congruent visual and somatic response properties. J Neurophysiol 79, 126-136.

Efron R \& Efron R (1969). What is perception? In Proceedings of the Boston Colloquium for the Philosophy of Science 1966/1968. Boston Studies in the Philosophy of Science, vol 4, ed. Cohen RS \& Wartofsky MW, pp. 137-173. Springer, Dordrecht.

Firth NC, Primativo S, Marinescu RV, Shakespeare TJ, Suarez-Gonzalez A, Lehmann M, Carton A, Ocal D, Pavisic I, Paterson RW, Slattery CF, Foulkes AJM, Ridha BH, Gil-Néciga E, Oxtoby NP, Young AL, Modat M, Cardoso MJ, Ourselin S, Ryan NS, Miller BL, Rabinovici GD, Warrington EK, Rossor MN, Fox NC, Warren JD, Alexander DC, Schott JM, Yong KXX \& Crutch SJ (2019). Longitudinal neuroanatomical and cognitive progression of posterior cortical atrophy. Brain 142, 2082-2095.

Golden HL, Nicholas JM, Yong KXX, Downey LE, Schott JM, Mummery CJ, Crutch SJ \& Warren JD (2015). Auditory spatial processing in Alzheimer's disease. Brain 138, 189-202.

Graff-Radford J, Yong KXX, Apostolova LG, Bouwman FH, Carrillo M, Dickerson BC, Rabinovici GD, Schott JM, Jones DT \& Murray ME (2021). New insights into atypical Alzheimer's disease in the era of biomarkers. Lancet Neurol 20, 222-234.
Hardy CJD, Yong KXX, Goll JC, Crutch SJ \& Warren JD (2020). Impairments of auditory scene analysis in posterior cortical atrophy. Brain 143, 2689-2695.

James M, Plant GT, Gordon T, Warrington EK \& Thames Valley Test Company (2001). Corvist: Cortical Vision Screening Test: Manual and Test Materials. Thames Valley Test Co., Bury St Edmunds.

Lackner J \& Dizio P (2005). Vestibular, proprioceptive, and haptic contributions to spatial orientation artificial gravity view project. Artic Annu Rev Psychol 56, 115-147.

Lehmann M, Crutch SJ, Ridgway GR, Ridha BH, Barnes J, Warrington EK, Rossor MN \& Fox NC (2011). Cortical thickness and voxel-based morphometry in posterior cortical atrophy and typical Alzheimer's disease. Neurobiol Aging 32, 1466-1476.

McKhann GM, Knopman DS, Chertkow H, Hyman BT, Jack CR, Kawas CH, Klunk WE, Koroshetz WJ, Manly JJ, Mayeux R, Mohs RC, Morris JC, Rossor MN, Scheltens $\mathrm{P}$, Carrillo MC, Thies B, Weintraub S \& Phelps CH (2011). The diagnosis of dementia due to Alzheimer's disease: recommendations from the National Institute on Aging-Alzheimer's Association workgroups on diagnostic guidelines for Alzheimer's disease. Alzheimers Dement 7, 263-269.

Mendez MF, Ghajaraniad M \& Perryman KM (2002). Posterior cortical atrophy: clinical characteristics and differences compared to Alzheimer's disease. Dement Geriatr Cogn Disord 14, 33-40.

Mittelstaedt H (1983). A new solution to the problem of the subjective vertical. Naturwissenschaften 70, 272-281.

Mittelstaedt H (1996). Somatic graviception. Biol Psychol 42, 53-74.

Nestor PJ, Caine D, Fryer TD, Clarke J \& Hodges JR (2003). The topography of metabolic deficits in posterior cortical atrophy (the visual variant of Alzheimer's disease) with FDG-PET. J Neurol Neurosurg Psychiatry 74, 1521-1529.

Pisella L, Sergio LE \& Blangero A (2009). Optic ataxia and the function of the dorsal stream: contributions to perception and action EPIPAGE 2 view project neural correlates underlying prism adaptation view project. Neuropsychologia 47, 3033-3044.

Ridgway GR, Omar R, Ourselin S, Hill DLG, Warren JD \& Fox NC (2009). Issues with threshold masking in voxel-based morphometry of atrophied brains. Neuroimage 44, 99-111.

Schott JMJM, Crutch SJ, Carrasquillo MM, Uphill J, Shakespeare TJ, Ryan NS, Yong KX, Lehmann M, Ertekin-Taner N, Graff-Radford NR, Boeve BF, Murray ME, Khan QUA, Petersen RC, Dickson DW, Knopman DS, Rabinovici GD, Miller BL, González AS, Gil-Néciga E, Snowden JS, Harris J, Pickering-Brown SM, Louwersheimer E, van der Flier WM, Scheltens P, Pijnenburg YA, Galasko D, Sarazin M, Dubois B, Magnin E, Galimberti D, Scarpini E, Cappa SF, Hodges JR, Halliday GM, Bartley L, Carrillo MC, Bras JT, Hardy J, Rossor MN, Collinge J, Fox NC \& Mead S (2016). Genetic risk factors for the posterior cortical atrophy variant of Alzheimer's disease. Alzheimers Dement 12, 862-871. 
Shakespeare TJ, Kaski D, Yong KXX, Paterson RW, Slattery CF, Ryan NS, Schott JM \& Crutch SJ (2015). Abnormalities of fixation, saccade and pursuit in posterior cortical atrophy. Brain 138, 1976-1991.

Tang-Wai DF, Graff-Radford NR, Boeve BF, Dickson DW, Parisi JE, Crook R, Caselli RJ, Knopman DS \& Petersen RC (2004). Clinical, genetic, and neuropathologic characteristics of posterior cortical atrophy. Neurology 63, 1168-1174.

Taylor-Clarke M, Kennett S \& Haggard P (2002). Vision modulates somatosensory cortical processing. Curr Biol 12, 233-236.

Van Engelen MPE, Gossink FT, De Vijlder LS, Meursing JRA, Scheltens P, Dols A \& Pijnenburg YAL (2020). End stage clinical features and cause of death of behavioral variant frontotemporal dementia and young-onset Alzheimer's disease. J Alzheimer's Dis 77, 1169-1180.

Vingerhoets RAA, De Vrijer M, Gisbergen JAM Van \& Medendorp WP (2009). Fusion of visual and vestibular tilt cues in the perception of visual vertical. Ebenholtz Benzschawel 101, 1321-1333.

Warren JD, Fletcher PD \& Golden HL (2012). The paradox of syndromic diversity in Alzheimer disease. Nat Rev Neurol 8, 451-464.

Warrington EK, James M \& Thames Valley Test Company (1991). The Visual Object and Space Perception Battery. Thames Valley Test Company, Bury St Edmunds.

Whitwell JL, Jack CR, Kantarci K, Weigand SD, Boeve BF, Knopman DS, Drubach DA, Tang-Wai DF, Petersen RC \& Josephs KA (2007). Imaging correlates of posterior cortical atrophy. Neurobiol Aging 28, 1051-1061.

World Medical Association (2013). World Medical Association declaration of Helsinki: ethical principles for medical research involving human subjects. JAMA 310, 2191-2194.

Yong KXX, McCarthy ID, Poole T, Suzuki T, Yang B, Carton AM, Holloway C, Papadosifos N, Boampong D, Langham J, Slattery CF, Paterson RW, Foulkes AJM, Schott JM, Frost C, Tyler N \& Crutch SJ (2018). Navigational cue effects in Alzheimer's disease and posterior cortical atrophy. Ann Clin Transl Neurol 5, 697-709.

Yong KXX, Shakespeare TJ, Cash D, Henley SMD, Nicholas JM, Ridgway GR, Golden HL, Warrington EK, Carton AM, Kaski D, Schott JM, Warren JD \& Crutch SJ (2014). Prominent effects and neural correlates of visual crowding in a neurodegenerative disease population. Brain 137, 3284-3299.

\section{Additional information}

\section{Data availability statement}

All data supporting presented results are available from the corresponding author upon reasonable request.

\section{Competing interests}

The authors declare no competing interests.

\section{Author contributions}

B.L.D. and S.J.C. designed research; B.L.D., D.O., A.P., M.J.B., D.C., D.K., S.J.C. and K.X.X.Y. acquired and interpreted data; B.L.D., D.O. and K.X.X.Y. analysed data; B.L.D., S.J.C. and K.X.X.Y. drafted the paper and all authors critically revised the paper. All authors have read and approved the final version of this manuscript and agree to be accountable for all aspects of the work in ensuring that questions related to the accuracy or integrity of any part of the work are appropriately investigated and resolved. All persons designated as authors qualify for authorship, and all those who qualify for authorship are listed.

\section{Funding}

This project was funded by an Alzheimer's Society project grant (AS-PG-14-022). The work was also supported by an Alzheimer's Research UK Senior Research Fellowship and ESRC/NIHR (ES/L001810/1) grant to S.C. D.O. has received funding from the European Research Council under the European Union's Seventh Framework Programme (FP7/2007-2013)/ERC grant agreement no. 616905. K.Y. is an Etherington PCA Senior Research Fellow and is funded by the Alzheimer's Society, grant number 453 (AS-JF-18-003). The Dementia Research Centre is supported by Alzheimer's Research UK, Brain Research Trust, and The Wolfson Foundation. This work was also supported by the NIHR Queen Square Dementia Biomedical Research Unit, and the NIHR UCL/H Biomedical Research Centre.

\section{Acknowledgements}

We are deeply indebted to all the research participants and their supporters without whom this work would not have been possible. We are also grateful to members of the PCA Support Group (www.raredementiasupport.org/ posterior-cortical-atrophy/) who inspired the study, and whose contributions are described in (Crutch et al. 2018). We are also grateful for support, clinical and technical advice from Prof. Jonathan Schott, Dr Aida Suarez Gonzalez, Dr Christopher Dakin, and Dr Rebecca St George.

\section{Keywords}

Alzheimer's disease, gravity, posterior cortical atrophy, spatial orientation, verticality perception

\section{Supporting information}

Additional supporting information can be found online in the Supporting Information section at the end of the HTML view of the article. Supporting information files available:

Peer Review History

Statistical Summary Document 